ARTICLE

\title{
Repetitive magnetic stimulation induces plasticity of inhibitory synapses
} Maximilian Lenz ${ }^{1}$, Christos Galanis ${ }^{1}$, Florian Müller-Dahlhaus ${ }^{2}$, Alexander Opitz $^{3,4}$, Corette J. Wierenga
Gábor Szabó ${ }^{6}$, Ulf Ziemann²,

Repetitive transcranial magnetic stimulation (rTMS) is used as a therapeutic tool in neurology and psychiatry. While repetitive magnetic stimulation (rMS) has been shown to induce plasticity of excitatory synapses, it is unclear whether rMS can also modify structural and functional properties of inhibitory inputs. Here we employed $10-\mathrm{Hz}$ rMS of entorhinohippocampal slice cultures to study plasticity of inhibitory neurotransmission on CA1 pyramidal neurons. Our experiments reveal a rMS-induced reduction in GABAergic synaptic strength (2-4 $\mathrm{h}$ after stimulation), which is $\mathrm{Ca}^{2+}$-dependent and accompanied by the remodelling of postsynaptic gephyrin scaffolds. Furthermore, we present evidence that $10-\mathrm{Hz}$ rMS predominantly acts on dendritic, but not somatic inhibition. Consistent with this finding, a reduction in clustered gephyrin is detected in CA1 stratum radiatum of rTMS-treated anaesthetized mice. These results disclose that rTMS induces coordinated $\mathrm{Ca}^{2+}$-dependent structural and functional changes of specific inhibitory postsynapses on principal neurons.

\footnotetext{
${ }^{1}$ Institute of Clinical Neuroanatomy, Neuroscience Center, Goethe-University, Frankfurt/Main 60590, Germany. ${ }^{2}$ Department of Neurology and Stroke and Hertie Institute for Clinical Brain Research, Eberhard-Karls-University, Tübingen 72076, Germany. ${ }^{3}$ Center for Biomedical Imaging and Neuromodulation, Nathan Kline Institute for Psychiatric Research, Orangeburg, New York 10962, USA. ${ }^{4}$ Center for the Developing Brain, Child Mind Institute, New York, New York 10022, USA. ${ }^{5}$ Division of Cell Biology, Faculty of Science, Utrecht University, Utrecht 3584 CH, The Netherlands. ${ }^{6}$ Laboratory of Molecular Biology and Genetics, Institute of Experimental Medicine, Budapest H1083, Hungary. ${ }^{7}$ Department of Neurophysiology, Medical Faculty, Ruhr-University, Bochum 44780, Germany. Correspondence and requests for materials should be addressed to A.V. (email: a.vlachos@med.uni-frankfurt.de).
} 
T he therapeutic potential of repetitive transcranial magnetic stimulation (rTMS) has been extensively studied in the context of brain diseases, such as addiction, depression, Parkinson's disease, schizophrenia and stroke ${ }^{1}$. Yet, the cellular and molecular mechanisms underlying rTMS-based therapies remain not well understood ${ }^{2,3}$. Thus, a better understanding of rTMS-induced neural plasticity is needed to optimize treatment protocols and to develop new diagnostic and therapeutic strategies using rTMS. Studies in suitable animal models or in appropriate in vitro preparations provide experimental approaches in this context, since electrophysiological, (live-cell) imaging and molecular biology techniques can be combined with genetic and pharmacologic methods to unravel how repetitive magnetic stimulation (rMS) induces neural plasticity ${ }^{4}$.

In our previous work we were able to demonstrate that rMS of mouse entorhinohippocampal slice cultures leads to long-lasting structural and functional changes of excitatory postsynapses, ${ }^{5,6}$. Using a $10-\mathrm{Hz}$ rMS protocol we observed a robust strengthening of excitatory inputs and the enlargement of dendritic spines on CA1 pyramidal neurons $(2-4 \mathrm{~h} \text { post-magnetic stimulation, pms })^{5}$. Since these changes were mediated by $\mathrm{N}$-methyl-D-aspartate receptors (NMDARs), the results of this earlier study indicated that rMS is capable of inducing long-term potentiation (LTP) of $\alpha$-amino-3hydroxy-5-methyl-4-isoxazolepropionic acid receptor-mediated synaptic transmission ${ }^{5}$. Furthermore, we recently showed that the effects of rMS on excitatory synapses occur predominantly on proximal dendrites of cultured CA1 pyramidal neurons ${ }^{6}$. Hence, rMS may induce plasticity of specific excitatory synapses of a neuron.

Considering recent experimental evidence, which indicates a crucial role for inhibitory synaptic transmission in cortical network function $^{7-9}$ and the suggestion that alterations in excitation and inhibition (E/I) balance underlie states of behavioural and cognitive dysfunction in brain diseases ${ }^{10-13}$, we here sought to determine the effects of the same $10-\mathrm{Hz}$ rMS protocol ${ }^{5,6}$ on structural and functional properties of inhibitory synapses. Gephyrin, the major postsynaptic scaffolding protein to which ionotropic $\gamma$-aminobutyric acid receptors $\left(\mathrm{GABA}_{\mathrm{A}} \mathrm{R}\right)$ anchor ${ }^{14-16}$, was used to test for rMS-induced structural changes of inhibitory postsynaptic sites on CA1 pyramidal neurons ${ }^{17}$. Our results disclose a reduction in inhibitory synaptic strength, which is accompanied by the destabilization of gephyrin clusters following rMS in vitro (2-4h after stimulation). These structural and functional changes require the activation of voltage-gated sodium channels (VGSCs), L-type voltage-gated calcium channels (LVGCCs) and NMDARs, and are not observed when calcineurin protein phosphatases are pharmacologically blocked. While the $\mathrm{Ca}^{2+}$-dependent cysteine protease (calpain) inhibitor MDL-28170 $(50 \mu \mathrm{M})^{18}$ has no effect in our experiments, the results of the present study corroborate the previously proposed gephyrinmediated $\mathrm{Ca}^{2+} /$ calcineurin-dependent tuning of inhibitory synapses, which accompanies LTP of excitatory synapses ${ }^{19}$. Notably, dendritic inhibition appears to be mainly affected in our experimental setting, suggesting that $10-\mathrm{Hz}$ rMS may not act equally on all inhibitory synapses of a neuron. Consistent with this finding, a reduction in gephyrin cluster sizes and numbers is observed in the dendritic layer, that is, CA1 stratum radiatum of $10-\mathrm{Hz}$ rTMS-treated anaesthetized mice. Together with our previous work ${ }^{5,6}$, we propose that $\mathrm{rMS}$ acts on network excitability and connectivity through the induction of coordinated, $\mathrm{Ca}^{2+}$-mediated changes of specific subsets of excitatory and inhibitory synapses on principal neurons.

\section{Results}

rMS of organotypic slice cultures. Slice cultures $(\geq 18$ days in vitro) containing the entorhinal cortex and the hippocampus were stimulated using a standard $70-\mathrm{mm}$ figure-of-eight coil (Fig. 1a) ${ }^{5,6}$. The electric field strength induced in the slice culture preparations was described using computational modelling $\left(27.3 \pm 3.3 \mathrm{~V} \mathrm{~m}^{-1}\right.$; Fig. 1b,c). Stimulated and non-stimulated (but otherwise equally treated) cultures were returned to the incubator and experimentally assessed at $2-4 \mathrm{~h} \mathrm{pms}$, which is the time period during which structural and functional changes of excitatory synapses are most prominently observed in our experimental setting.

rMS induces changes in inhibitory synaptic transmission. To determine rMS-induced changes in inhibitory synaptic strength we patched individual CA1 pyramidal neurons and recorded miniature inhibitory postsynaptic currents (mIPSCs) in whole-cell voltage-clamp mode from non-stimulated control and stimulated cultures (Fig. 1d-f). Recordings were performed at a holding potential of $-80 \mathrm{mV}$ in the presence of tetrodotoxin (TTX; $0.5 \mu \mathrm{M}$ ), which inhibits VGSCs, as well as inhibitors of NMDARs (D(-)-2-amino-5-phosphonovaleric acid, AP5, 10 $\mu \mathrm{M})$ and $\alpha$-amino-3-hydroxy-5-methyl-4-isoxazolepropionic acid receptors (6-cyano-7-nitroquinoxaline-2,3-dione, CNQX, $10 \mu \mathrm{M}$ ). Hence, we assessed properties of $\mathrm{GABA}_{\mathrm{A}} \mathrm{R}$-mediated currents evoked by stochastic release of GABA from presynaptic terminals (Fig. 1f), which could be blocked by the $\mathrm{GABA}_{\mathrm{A}} \mathrm{R}$ inhibitor SR95531 $(10 \mu \mathrm{M})$ or bicuculline-methiodide $(50 \mu \mathrm{M} ;$ c.f., Fig. 2a). In these experiments a significant reduction in mIPSC amplitudes was observed after rMS, while mIPSC frequencies were not significantly changed (Fig. 1g,h). Since the amplitude of mIPSCs is considered to reflect postsynaptic strength, we conclude that rMS in vitro induces a reduction in $\mathrm{GABA}_{\mathrm{A}} \mathrm{R}$-mediated synaptic transmission on CA1 pyramidal neurons $2-4 \mathrm{~h} \mathrm{pms.}$

rMS has no major effect on tonic inhibition. We then tested for the effects of $10-\mathrm{Hz}$ rMS on extrasynaptic $\mathrm{GABA}_{\mathrm{A}} \mathrm{R}$ conductance, which is mediated by ambient GABA in the extracellular space (Fig. 2). A different set of cultures was stimulated and individual CA1 pyramidal neurons were patched $2-4 \mathrm{~h}$ pms in presence of TTX, AP5 and CNQX. After obtaining stable baseline recording, GABA $(5 \mu \mathrm{M})$-containing extracellular solution was washed into the recording chamber, followed by bicuculline-methiodide $(50 \mu \mathrm{M})$-containing extracellular solution to block $\mathrm{GABA}_{\mathrm{A}} \mathrm{Rs}$ at the end of each experiment (Fig. 2a). No significant difference between the two groups was observed in (1) the baseline (control: $-191.2 \pm 12.1 \mathrm{pA}, n=8$ neurons from eight cultures; rMS: $-187.5 \pm 13.6 \mathrm{pA}, n=6$ neurons from six cultures; $P=0.57$; Mann-Whitney test), (2) the GABA-mediated shift or (3) the bicuculline-induced change in tonic $\mathrm{GABA}_{\mathrm{A}} \mathrm{R}$ currents (Fig. $2 \mathrm{~b}$ ). Notably, we were able to confirm a significant reduction in mIPSC amplitudes after rMS in the baseline recordings (mIPSC amplitude, control: $34.4 \pm 1.6 \mathrm{pA}, n=8$ neurons from eight cultures; rMS: $28.4 \pm 1.7 \mathrm{pA}, n=6$ neurons from six cultures; $P<0.05$; Mann-Whitney test; mIPSC frequency, control: $9.3 \pm 0.8 \mathrm{~Hz}, \quad n=8 ; \quad$ rMS: $\quad 7.6 \pm 0.5 \mathrm{~Hz}, \quad n=6 ; \quad P=0.14$; Mann-Whitney test; internal solution containing higher $\left[\mathrm{Cl}^{-}\right]$ compared with Fig. 1). Consistent with these findings we did not detect a significant difference in $\mathrm{GABA}_{\mathrm{A}} \mathrm{R} \alpha 5$ immunostainings between stimulated and non-stimulated cultures $3 \mathrm{~h}$ after rMS (Supplementary Fig. 1; in line with previous work clustered $\mathrm{GABA}_{\mathrm{A}} \mathrm{R} \alpha 5$ staining was observed ${ }^{20,21}$ ). Although these experiments do not fully exclude the possibility that rMS affects tonic inhibition, the negative immunostaining results for $\mathrm{GABA}_{\mathrm{A}} \mathrm{R} \alpha 5$ (c.f. Supplementary Fig. 1) and the mIPSC results (c.f., Fig. 1) prompted us to focus on rMS-induced changes in phasic, that is, synaptic inhibitory neurotransmission. 
a

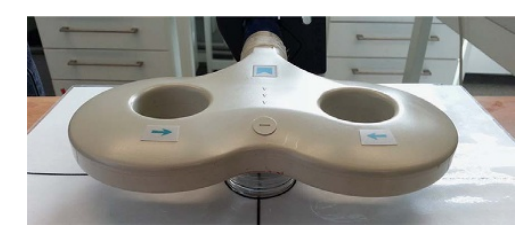

b

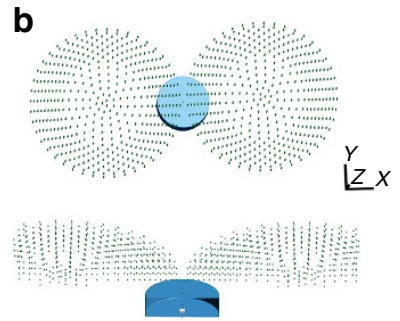

C

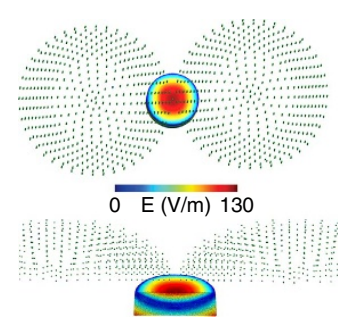

d
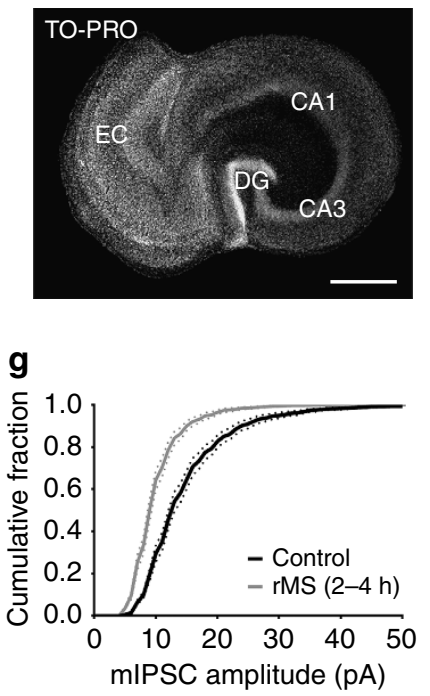

e

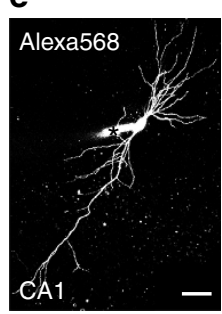

f

Control

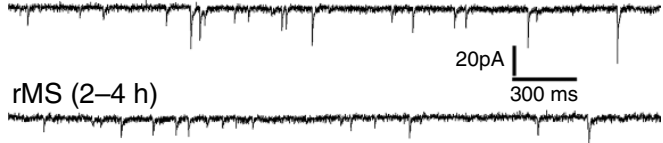

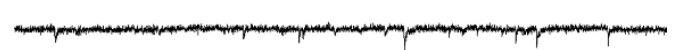

g

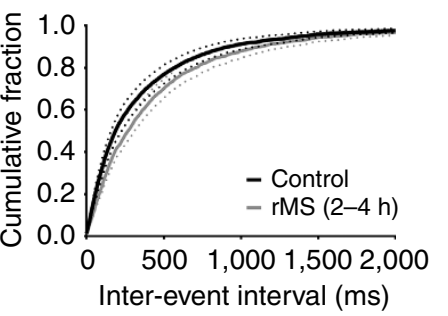

h

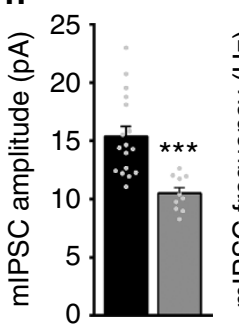

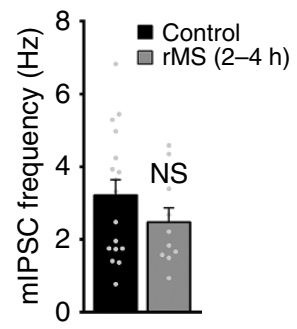

Figure 1 | rMS of mouse entorhinohippocampal slice cultures induces a reduction in inhibitory synaptic transmission. (a) A photograph illustrating the experimental setting. Slice cultures (Petri dish below the coil) are stimulated with a standard 70-mm outer wing diameter figure-of-eight coil. Distance from coil, position and orientation of cultures within the magnetic field are kept constant in all experiments $\mathrm{s}^{5,6}$. (b,c) The electric field induced by rMS in vitro was described based on a magnetic dipole model of the 70-mm figure-of-eight coil ${ }^{62}$. A finite element method model was created with the dimensions of the bath (blue) and tissue (grey) adapted to the in vitro setting. The coil was positioned $1 \mathrm{~cm}$ above the tissue (b). The electric field induced in our experimental setting was estimated to be $27.3 \pm 3.3 \mathrm{Vm}^{-1}$ in the tissue (c), which is within the lower range of clinical TMS motor-evoked potential threshold amplitudes of 30-130 V m${ }^{-1}$ (ref. 68). (d) Entorhinohippocampal slice culture stained with TO-PRO nuclear stain. All cultures were oriented in the same direction. Four to six cultures were stimulated at the same time (EC, entorhinal cortex; DG, dentate gyrus). Scale bar, $500 \mu \mathrm{m}$. (e) Patched CA1 pyramidal neuron filled with Alexa568. Asterisk indicates the Alexa568 (10 $\mu \mathrm{M})$ containing tip of the patch pipette. Scale bar, $50 \mu \mathrm{m}$. (f) Sample traces of mIPSCs recorded from CA1 pyramidal neurons in non-stimulated control cultures and cultures $2-4 \mathrm{~h}$ after rMS. (g,h) Cumulative distributions of mIPSC amplitudes and interevent intervals $(\mathbf{g})$. A significant reduction in the mean mIPSC amplitude but not the mean mIPSC frequency is observed $2-4 \mathrm{~h}$ after stimulation (h; control, $n=17$ neurons; $r M S, n=10$ neurons from five cultures each; Mann-Whitney test). Individual data points are indicated by gray dots. Values represent mean \pm s.e.m. ( ${ }^{\star \star \star} P<0.001 ; n s$, not significant differences).

rMS reduces size and number of gephyrin clusters. Accordingly, we tested whether molecular changes of inhibitory postsynapses accompany the rMS-induced functional changes in mIPSC amplitudes (Fig. 3). Slice cultures were immunostained for gephyrin, that is, the major postsynaptic scaffolding protein to which synaptic $\mathrm{GABA}_{\mathrm{A}}$ Rs anchor ${ }^{14-16}$ (Fig. 3a). Previous work demonstrated a close correlation between inhibitory synaptic strength, that is, mIPSC amplitudes and the size and stability of gephyrin clusters in CA1 stratum radiatum of entorhinohippocampal slice cultures ${ }^{17}$. Indeed, $3 \mathrm{~h}$ after rMS a significant reduction in gephyrin cluster sizes and numbers was observed in the stratum radiatum (Fig. $3 b$ ), indicating that rMS in vitro induces coordinated structural and functional changes of inhibitory postsynaptic sites.

rMS has no major effect on gephyrin expression. To test whether the observed changes in gephyrin cluster properties reflect rMS-induced changes in gephyrin expression, tissue mechanically isolated from slice cultures containing the
CA1 region was subjected to western blot and quantitative polymerase chain reaction (qPCR) analyses (Supplementary Fig. 2). In these experiments no significant difference was observed between the two groups (stimulated versus nonstimulated cultures), suggesting that rMS-induced changes in gephyrin clusters (at $3 \mathrm{~h}$ pms) cannot be trivially explained by a strong reduction in the expression of total gephyrin protein or mRNA.

rMS destabilizes GFP-gephyrin clusters. We then hypothesized that rMS-induced changes in gephyrin turn-over could explain our findings. Hence, the effects of rMS on dynamic properties of gephyrin clusters were assessed in slice cultures prepared from Thy1-GFP/gephyrin mice ${ }^{17}$. In these mice gephyrin tagged with enhanced green fluorescent protein (GFP) is expressed in a subset of principal neurons (Fig. 3c) ${ }^{22}$. To test for changes in the exchange rate of gephyrin, we determined fluorescence recovery after photobleaching (FRAP) of GFP-gephyrin clusters in the stratum radiatum of rMS-treated cultures versus non-stimulated 
a

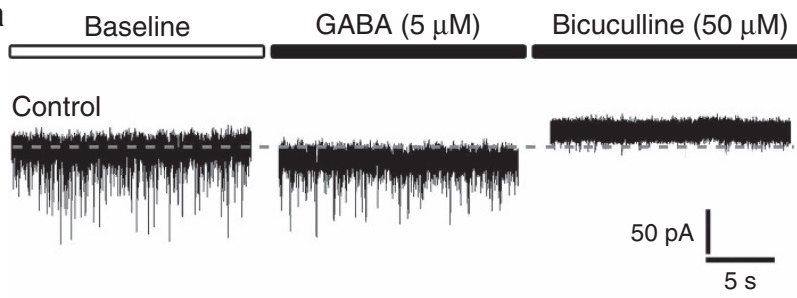

rMS (2-4 h)
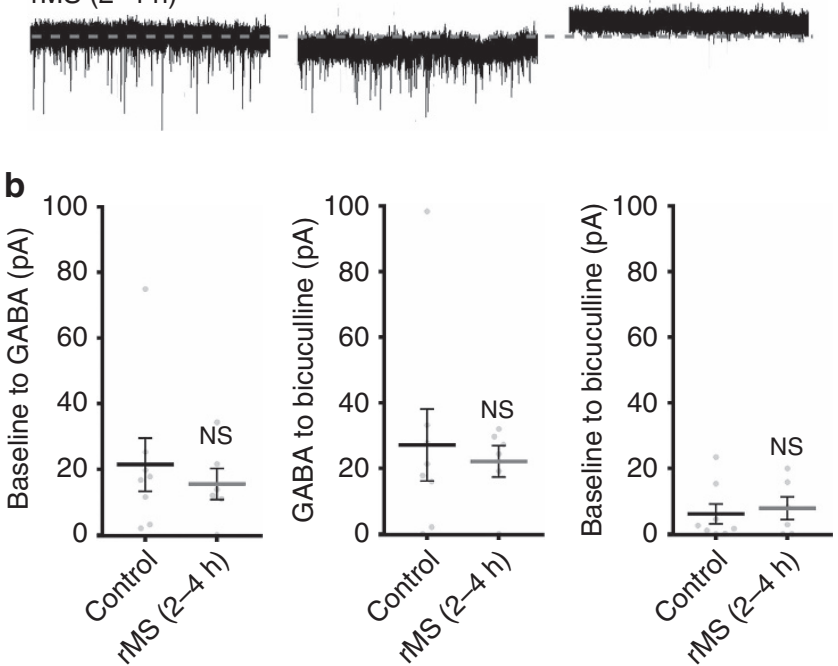

Figure 2 | Tonic GABA $_{A} R$ conductance is not affected after rMS. (a) Sample traces depicting GABA- $(5 \mu \mathrm{M})$ and bicuculline-methiodide (50 $\mu \mathrm{M})$ induced shift in tonic $G A B A_{A} R$-mediated currents recorded from $C A 1$ pyramidal neurons in non-stimulated control and stimulated slice cultures (2-4 $\mathrm{h}$ after $\mathrm{rMS}$ ). Baseline currents were not significantly different between the two groups (control: $191.2 \pm 12.1 \mathrm{pA}$; rMS: $187.5 \pm 13.6 \mathrm{pA} ; P=0.57$; control, $n=8$ neurons; rMS, $n=6$ neurons; one cell per culture; MannWhitney test). (b) The amplitude of GABA- or bicuculline-methiodideinduced shifts in tonic $G A B A_{A} R$ currents of $C A 1$ pyramidal neurons is not significantly different between stimulated and non-stimulated slice cultures (control, $n=8$ neurons; rMS, $n=6$ neurons; one cell per culture; MannWhitney test). Individual data points are indicated by grey dots. Values represent mean \pm s.e.m. (ns, not significant differences).

control cultures ${ }^{17}$. After a 15 -min baseline recording, individual GFP-gephyrin clusters were photobleached $(<5 \%$ of initial fluorescence intensity). The recovery, that is, reappearance of GFP-fluorescence was then followed for $80 \mathrm{~min}$ at 5-min intervals (Fig. 3d). In non-stimulated control cultures $30 \%$ of the averaged pre-bleach GFP-gephyrin fluorescence recovered within $\sim 40 \mathrm{~min}$. In contrast, following $\mathrm{rMS} 30 \%$ recovery was reached already $\sim 7.5 \mathrm{~min}$ after the bleach, and after $80 \mathrm{~min}>60 \%$ of the pre-bleach signal was detected (Fig. 3e,f). These experiments disclosed a significantly faster recovery of GFP-gephyrin clusters $2-4 \mathrm{~h}$ following $\mathrm{rMS}$ in vitro (Fig. 3f; for averaged 70-80 min values; pooled non-stimulated control data). Taken together, our FRAP experiments showed that the re-incorporation of GFPgephyrin into individual gephyrin clusters is accelerated after rMS. Since GFP-gephyrin clusters of comparable pre-bleach intensity were assessed in these experiments (control: $1.0 \pm 0.02$, $n=53$ clusters from eight cultures; rMS: $1.1 \pm 0.03, n=35$ clusters from six cultures; values normalized to control; $P=0.15$; Mann-Whitney test), we attribute this acceleration to an increased turnover, that is, rMS-induced destabilization of postsynaptic gephyrin scaffolds ${ }^{17} 2-4 \mathrm{~h}$ following magnetic stimulation.
rMS reduces size and number of $\mathrm{GABA}_{\mathrm{A}} \mathrm{R} \alpha 2$ clusters. To link the described changes in gephyrin cluster properties to our electrophysiological recordings, we stained stimulated and non-stimulated slice cultures for $\mathrm{GABA}_{\mathrm{A}} \mathrm{R}$ subunit $\alpha 2$, which anchor synaptic $\mathrm{GABA}_{\mathrm{A}} \mathrm{Rs}$ to gephyrin ${ }^{23}$ (Fig. 4a). Indeed, a significant reduction in $\mathrm{GABA}_{\mathrm{A}} \mathrm{R} \alpha 2$ cluster sizes and numbers was observed in the stratum radiatum $3 \mathrm{~h}$ after stimulation in these experiments (Fig. 4b).

Paired recordings disclose rMS effects on synaptic inhibition. To further characterize rMS-induced changes in inhibitory synaptic transmission at the level of individual connected neurons, paired recordings were carried out. In this set of experiments, slice cultures prepared from GAD65-GFP mice ${ }^{24}$ were used (Fig. 5). Interneurons mainly projecting their axons on dendrites of CA1 neurons can be readily identified by the GFP signal in these preparation $\mathrm{s}^{22}$ (Fig. 5a). GFP-expressing interneurons (in the stratum radiatum or at the border to the stratum lacunosum moleculare) and CA1 pyramidal neurons were patched at the same time, and the connectivity between pairs of neurons was probed in stimulated and nonstimulated cultures (Fig. 5b). Neurons were considered connected if $>5 \%$ of presynaptic action potentials evoked time-locked postsynaptic inward current responses (Fig. 5c; up to 50 action potentials induced at $0.1 \mathrm{~Hz}$ ). In non-stimulated control cultures the probability to find connected pairs was $\sim 60 \%$ (Fig. $5 \mathrm{~d}$ ). Following rMS, a marked decrease (down to $\sim 25 \%$ ) in the probability to find connected neurons was observed (Fig. 5d). In addition, the mean amplitude of successfully evoked IPSCs in response to single presynaptic action potentials was significantly decreased following rMS (Fig. 5e). Interestingly, a marked increase in the percentage of action potentials not successfully evoking postsynaptic current responses in connected pairs was detected 2-4 h after rMS (Fig. 5f; synaptic failure rate). Because paired-pulse and short-term plasticity were not significantly changed after rMS (Fig. 5g,h), we attribute the observed changes in failure rates to a decrease in the number of functional synapses between inhibitory interneurons and CA1 neurons, rather than to changes in the presynaptic release probability. This suggestion is in line with our findings on reduced gephyrin and $\mathrm{GABA}_{\mathrm{A}} \mathrm{R} \alpha 2$ cluster numbers in the stratum radiatum after rMS (Figs 3 and 4), while mIPSC frequencies were not significantly reduced (Fig. 1g,h). Hence, although rMS-induced presynaptic changes cannot be excluded, these experiments confirmed that rMS leads to profound changes in the connectivity of inhibitory networks, that is, fewer, less efficient and weaker inhibitory synapses on CA1 pyramidal neurons.

VGSC activity is required for rMS-induced plasticity. Which signals mediate the rMS-induced reduction in inhibitory synaptic transmission? Because rMS is expected to depolarize neurons and to induce action potentials ${ }^{25}$, we first tested whether the activation of VGSCs is required for the rMS-induced structural and functional remodelling of inhibitory postsynapses (Table 1; TTX). To this end, slice cultures were stimulated in the presence of TTX $(2 \mu \mathrm{M} \text {; washed out immediately after stimulation })^{6}$ and mIPSC recordings, gephyrin immunostainings, GFP-gephyrin FRAP experiments and $\mathrm{GABA}_{\mathrm{A}} \mathrm{R} \alpha 2$ immunostainings were repeated (c.f., Figs 1,3 and 4). Indeed, in these experiments the effects of rMS in vitro on structural and functional properties of inhibitory postsynapses were completely blocked. We conclude that VGSC activity is required for rMS-induced plasticity of inhibitory postsynaptic sites.

NMDAR and L-VGCC activities mediate rMS-induced plasticity. Considering the previously proposed $\mathrm{Ca}^{2+}$-dependent tuning of inhibitory synapses, which accompanies LTP of excitatory 
a
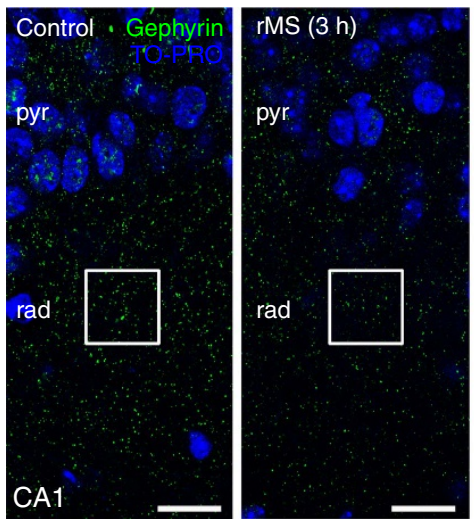

C

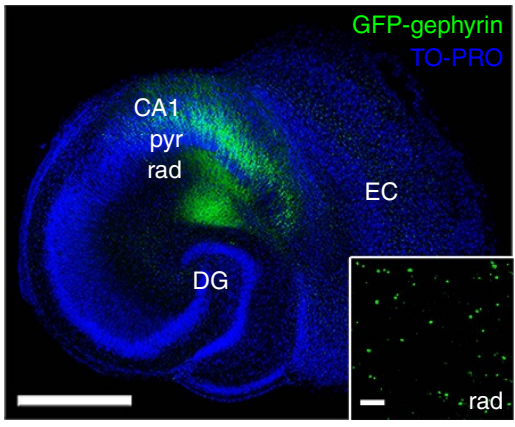

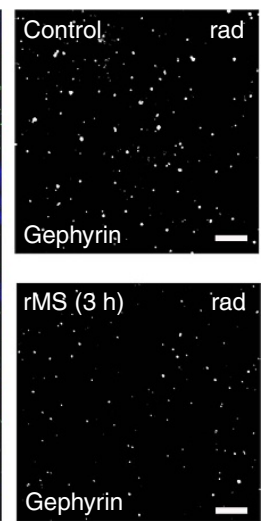
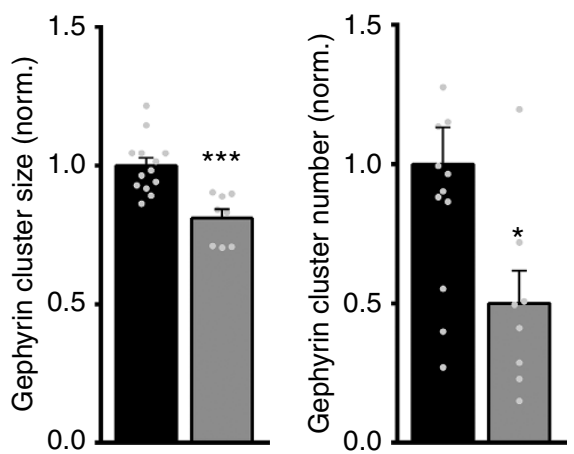

Control d
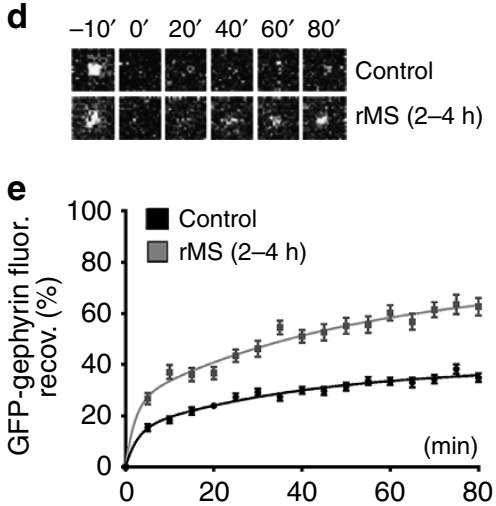

$\mathbf{f}$

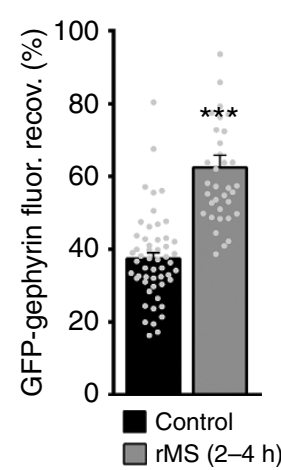

Figure 3 | rMS induces changes in gephyrin cluster properties. (a) Stimulated and non-stimulated cultures stained for endogenous gephyrin. Cluster sizes and numbers were assessed in the CA1 stratum radiatum (rad). Blue, TO-PRO nuclear stain; pyr, stratum pyramidale. Scale bars, $30 \mu \mathrm{m}$ (at higher magnification, $4 \mu \mathrm{m}$ ). (b) A reduction in the mean gephyrin cluster size and number is observed after rMS. Values normalized to non-stimulated control cultures (control, $n=13$ cultures; rMS, $n=8$ cultures; averaged data from three visual fields per culture; Mann-Whitney test; two data points outside the axis limits). (c) Overview of a slice culture prepared from a Thy1-GFP/gephyrin mouse. GFP-gephyrin expression (green) is regularly seen in area CA1 in these preparations. FRAP of individual GFP-gephyrin clusters was assessed in the stratum radiatum (rad) of area CA1. Blue, TO-PRO nuclear stain; pyr, stratum pyramidale; DG, dentate gyrus; EC, entorhinal cortex. Scale bar, $500 \mu \mathrm{m}$ (inset shows GFP-gephyrin clusters at higher magnification; scale bar, $4 \mu \mathrm{m}$ ). (d) FRAP images of single GFP-gephyrin clusters in the CA1 stratum radiatum after bleaching at 0 min. Series of images taken from a non-stimulated control culture and a culture 2-4h after rMS. (e,f) Quantitative evaluation of FRAP experiments. (e) Accelerated GFP-gephyrin FRAP is observed following rMS in vitro. (f) Group data for averaged 70-80 min FRAP values in non-stimulated and stimulated slice cultures (control, $n=53$ clusters from eight cultures; rMS, $n=35$ clusters from six cultures; $5-12$ clusters bleached per culture; Kruskal-Wallis test followed by Dunn's post hoc test; one data point in $\mathbf{f}$ outside the axis limits; pooled non-stimulated control data). Individual data points are indicated by grey dots. Values represent mean \pm s.e.m. $\left({ }^{\star} P<0.05\right.$; ${ }^{\star \star \star} P<0.001$; ns, not significant differences).

synapses ${ }^{19}$ and our earlier findings on the role of NMDARs and L-VGCCs in rMS-induced potentiation of excitatory synapses ${ }^{5,6}$, we next tested whether pharmacological inhibition of NMDARs with AP5 $(50 \mu \mathrm{M})$ or blocking L-VGCCs with Nifedipin $(20 \mu \mathrm{M})$ hampers rMS-induced plasticity of inhibitory synapses (Table 1; AP5 and Nifedipin; inhibitors were applied only during rMS and washed out immediately after the end of stimulation). Except for a reduction in gephyrin cluster numbers in AP5-treatedstimulated cultures, we observed that mIPSC amplitudes, gephyrin clusters, GFP-gephyrin FRAP and $\mathrm{GABA}_{\mathrm{A}} \mathrm{R} \alpha 2$ cluster sizes and numbers in the stratum radiatum were comparable in stimulated and non-stimulated slice cultures. We conclude from these experiments that rMS induces $\mathrm{Ca}^{2+}$-dependent tuning of inhibitory inputs, that is, a reduction in inhibitory synaptic strength, which involves the activation of NMDARs and L-VGCCs during stimulation.

Calcineurin mediates rMS-induced plasticity. To test for possible downstream signalling pathways, we next blocked $\mathrm{Ca}^{2+}$ dependent, non-lysosomal cysteine proteases (calpain) or calcineurin protein phosphatases. Previous work showed that the degradation/destabilization of gephyrin clusters can be mediated by these $\mathrm{Ca}^{2+}$-dependent signalling pathways ${ }^{18,19}$. Accordingly, cultures were stimulated in presence of MDL-28170 $(50 \mu \mathrm{M})$ to block calpain or Cyclosporin A $(2 \mu \mathrm{M})$ to block calcineurin. In these experiments, pharmacologic inhibition of enzymatic activity was continued after stimulation. We found that MDL-28170 did not block the rMS-induced weakening of inhibitory inputs (Table 1; MDL-28170; changes in $\mathrm{GABA}_{\mathrm{A}} \mathrm{R} \alpha 2$ cluster numbers did not reach the level of significance), but Cyclosporin $\mathrm{A}$ prevented the reduction in mIPSC amplitude, the remodelling of gephyrin clusters, the destabilization of GFP-gephyrin clusters and reduction in $\mathrm{GABA}_{\mathrm{A}} \mathrm{R} \propto 2$ cluster sizes and numbers following rMS in vitro (Table 1; Cyclosporin A; a slight increase in gephyrin cluster sizes was observed in Cyclosporin A-treated-stimulated cultures). Taken together, these experiments suggest that rMS remodels inhibitory synapses in a calcineurin-dependent manner.

rMS does not affect all inhibitory synapse equally. Since our recent work revealed that rMS in vitro acts on specific subsets of 
a

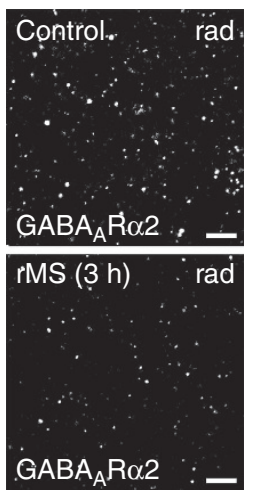

b

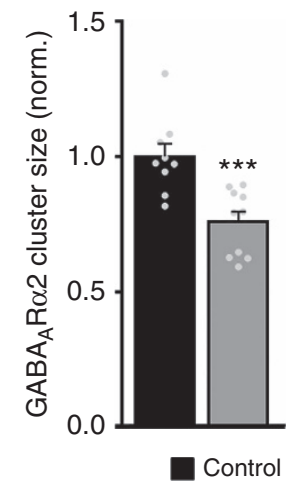

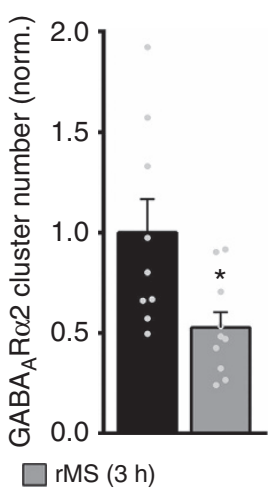

Figure 4 | rMS induces a reduction in $\mathbf{G A B A}_{\mathrm{A}}$ receptor $\alpha \mathbf{2}$ clusters. (a) Stimulated and non-stimulated cultures were stained for $\mathrm{GABA}_{\mathrm{A}}$ receptor subunit $\alpha 2\left(G A B A_{A} R \alpha 2\right)$, which anchors $G A B A_{A}$ Rs to postsynaptic gephyrin scaffolds. Cluster sizes and numbers were assessed in the CA1 stratum radiatum (rad). Scale bar, $3 \mu \mathrm{m}$. (b) rMS induces a decrease in the mean $\mathrm{GABA}_{\mathrm{A}} \mathrm{R} \alpha 2$ cluster size and number. Values normalized to non-stimulated control cultures (control, $n=9$ cultures; $r M S, n=10$ cultures; averaged data from three visual fields per culture; Mann-Whitney test). Individual data points are indicated by grey dots. Values represent mean \pm s.e.m. $\left({ }^{\star} P<0.05\right.$; ${ }^{* \star *} P \leq 0.001 ; \mathrm{ns}$, not significant differences).

excitatory inputs ${ }^{6}$, and because spatially separate inhibitory inputs are considered to mediate diverse neuronal functions $9,26,27$, we next tested whether rMS in vitro acts differentially on distinct subsets of inhibitory postsynapses. At this point, we focused on comparing somatic versus dendritic inhibition. First, slice cultures were double-stained for gephyrin and parvalbumin, which is a marker for inhibitory interneurons that project their axons mainly within the pyramidal cell layer, that is, the layer in which the cell bodies of CA1 pyramidal neurons are located (Fig. 6a). In these experiments, we assessed gephyrin clusters that were associated with parvalbumin-positive structures in the stratum pyramidale (Fig. 6b) and found no change in cluster sizes at $3 \mathrm{~h}$ following rMS (Fig. 6c). Analysis of all clusters in the stratum pyramidale provided similar results (Fig. 6d), while in the same set of cultures a reduction in the mean gephyrin cluster size and number was observed in the stratum radiatum (Fig. 6e; all clusters analysed). These experiments indicate that rMS may not affect somatic inhibition while reducing dendritic inhibition.

rMS does not affect somatic inhibition. To verify these results, paired recordings of parvalbumin-positive interneurons and CA1 pyramidal neurons were employed, similar to the experiments described in Fig. 5. To readily identify parvalbumin-expressing interneurons, slice cultures prepared from GAD67-GFP 28 a

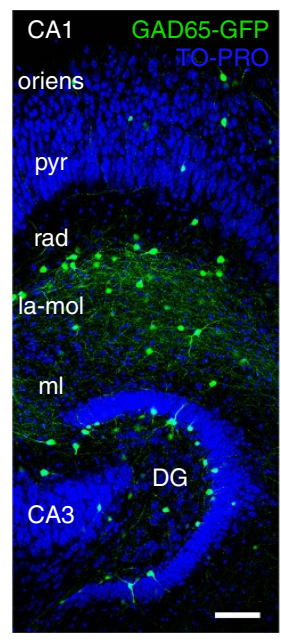

b

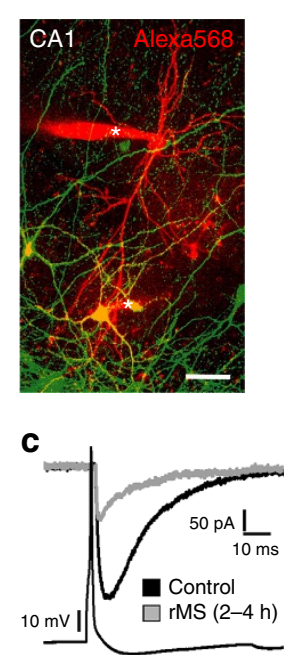

d
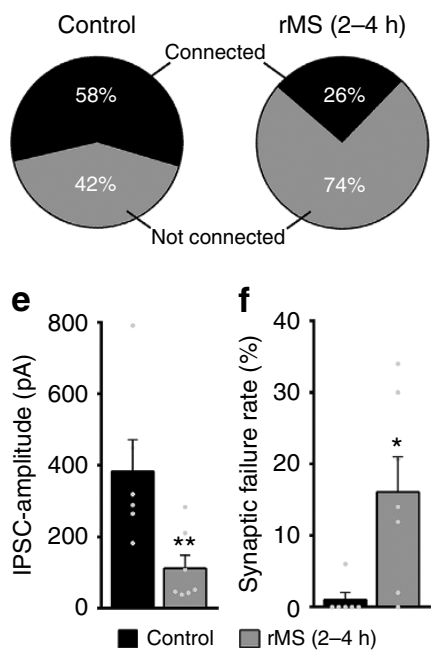

g

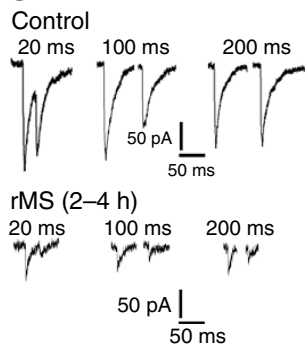

h

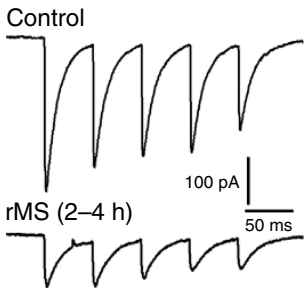

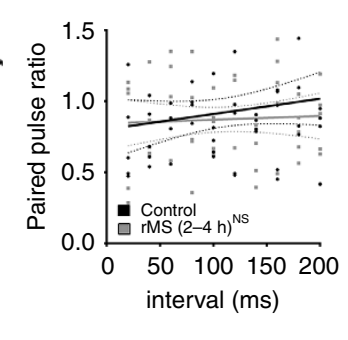

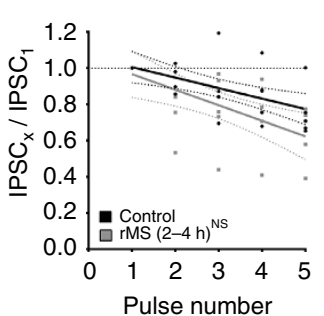

Figure 5 | Paired recordings disclose changes in inhibitory synaptic strength, efficacy and connectivity following rMS. (a) Overview of a slice culture prepared from GAD65-GFP mice. Note GFP-expressing interneurons in the stratum radiatum (rad) and lacunosum moleculare (la-mol) of CA1.

Occasionally, GFP-expressing interneurons are found in the stratum pyramidale (pyr) and in stratum oriens (oriens). Blue, TO-PRO nuclear stain. Scale bar, $100 \mu \mathrm{m}$. (b) Example of simultaneously patched neurons filled with Alexa568 $(10 \mu \mathrm{M})$. GFP-expressing interneurons are patched in rad or at the border between rad and la-mol. Asterisks indicate positions of the tips of Alexa568-filled patch pipettes. Scale bar, $50 \mu \mathrm{m}$. (c) Averaged responses of successfully evoked time-locked postsynaptic currents from CA1 neurons in control (black trace) and stimulated slice cultures (grey trace). Up to 50 action potentials $(45 \pm 3$ ) were induced at $0.1 \mathrm{~Hz}$ in presynaptic GFP-expressing interneurons while recording from postsynaptic CA1 neurons. (d-f) A decrease in the percentage of connected pairs and evoked GABA $R$-mediated IPSC amplitudes was observed, while the percentage of transmission failure rate was markedly increased after rMS (2-4h pms; control; $n=6$ connected pairs in four cultures, one value (1,196 pA with $0 \%$ failure rate) excluded from analysis; 7 of 12 probed pairs connected; rMS, $n=7$ connected pairs in four cultures; 7 of 27 probed pairs connected; Mann-Whitney test). (g) Sample traces illustrating paired-pulse protocols at different interpulse intervals (left). No significant difference between non-stimulated and stimulated cultures in pairedpulse depression is observed (control, $n=5$ cells in four cultures; rMS (2-4h), $n=5$ cells in four cultures, linear regression fit, Kruskal-Wallis test followed by Dunn's post hoc test; six data points outside the axis limits). (h) Sample traces illustrating short-term plasticity (five action potentials at $20 \mathrm{~Hz}$ ). The amplitude of each consecutive inhibitory postsynaptic current was normalized to the amplitude of the first response. No significant difference between nonstimulated and stimulated cultures (control, $n=5$ cells in four cultures; rMS ( $2-4 h$ ), $n=5$ cells in four cultures, linear regression fit, Kruskal-Wallis test followed by Dunn's post hoc test; one data points outside the axis limits). Individual data points are indicated by grey dots. Values represent mean \pm s.e.m. $\left({ }^{\star} P<0.05 ;{ }^{\star} P<0.01 ;\right.$ ns, not significant differences). 


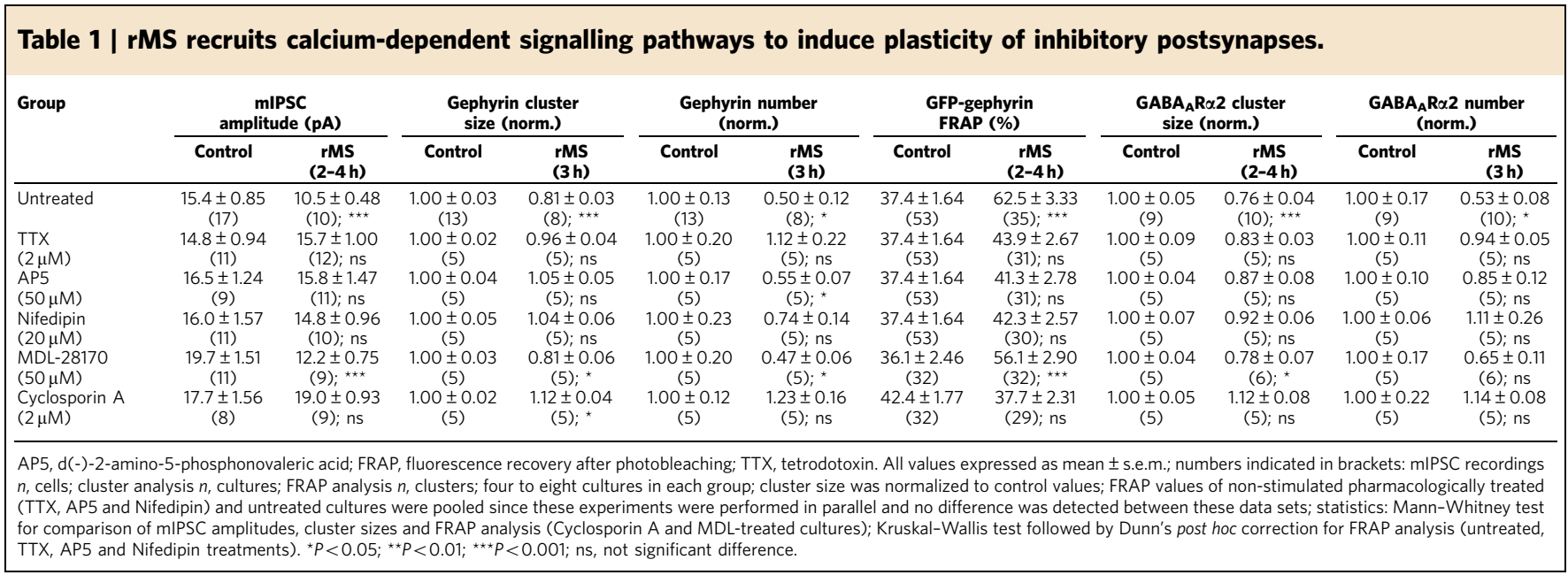

transgenic mice were used. After confirming the identity of GFP-expressing neurons in the CA1 stratum pyramidale by immunostaining for parvalbumin (Fig. 6f; different set of cultures), pairs of neurons were recorded to determine connectivity, as well as strength and efficacy of CA1 somatic inhibitory neurotransmission (Fig. $6 g-j$ ). At the end of the experiments, the fast spiking property of the patched GFP-positive interneuron was confirmed (Supplementary Fig. 3). No significant difference was observed between stimulated and non-stimulated slice cultures in these experiments (Fig. 6i,j; paired-pulse protocol and short-term plasticity given in Supplementary Fig. 3). Of note, in the same set of recorded CA1 neurons a significant reduction in the mean amplitude of spontaneous IPSCs was detected (sIPSC amplitude, control: $18.8 \pm 1.0 \mathrm{pA}, n=8$ neurons from four cultures; rMS: $14.5 \pm 1.3 \mathrm{pA}, n=9$ neurons from three cultures; $P<0.05$; sIPSC frequency, control: $6.3 \pm 1.3 \mathrm{~Hz}, n=8$; rMS: $5.2 \pm 0.7 \mathrm{~Hz}, n=9$; $P=0.66$; Mann-Whitney test). We conclude from these experiments that $10-\mathrm{Hz}$ rMS in vitro does not exert its major effects by changing somatic inhibition.

GABA uncaging reveals rMS effects on dendritic inhibition. To provide further support for the differential effects of rMS on inhibitory postsynapses, rMS-induced functional changes in somatic versus dendritic inhibition were determined in the same set of neurons using flash photolysis of caged-GABA (Fig. 7; 'GABA-uncaging experiments'). This technique enabled us to separately stimulate somatic versus dendritic $\mathrm{GABA}_{\mathrm{A}} \mathrm{Rs}$ while recording uncaging-evoked IPSCs from the soma of individual CA1 pyramidal neurons (Fig. 7a,b). Care was taken to accurately position the laser beam for locally releasing GABA to target either the soma or proximal part of the apical principal dendrite of the Alexa-filled postsynaptic neuron (Fig. 7a). For each recorded neuron, Ruthenium-bipyridine-triphenylphosphine caged GABA (RuBi-GABA)-uncaging-evoked inward current responses to five consecutive laser-light pulses at $0.1 \mathrm{~Hz}$ in each region (somatic versus dendritic) were averaged (Fig. $7 \mathrm{~b}$ ). The uncaging responses were blocked by the $\mathrm{GABA}_{\mathrm{A}} \mathrm{R}$ inhibitor SR95531 and did not occur when the laser beam was directed on the neurophil in the neighbourhood of the patched CA1 pyramidal neurons (that is, $>10 \mu \mathrm{m}$ away from the soma or closest dendrite) ${ }^{29}$. Responses to RuBi-GABA uncaging at somatic locations were not significantly different in the two groups, whereas dendritic GABA responses were markedly decreased in the rMS group (Fig. 7c). Since these experiments were performed in the presence of TTX, AP5 and CNQX, we were also able to record mIPSC events from a different set of neurons in this round of experiments (Fig. 7d).
Thus, we verified once more that the mean mIPSC amplitude is reduced $2-4 \mathrm{~h}$ after $\mathrm{rMS}$ (Fig. $7 \mathrm{~d}$; internal solution containing higher $\left[\mathrm{Cl}^{-}\right]$compared with Fig. 1). Together with our paired recordings and the gephyrin immunostaining (Figs 5 and 6), we conclude that $10-\mathrm{Hz}$ rMS in vitro predominantly affects dendritic (but not somatic) $\mathrm{GABA}_{\mathrm{A}} \mathrm{R}$-mediated synaptic inhibition.

rTMS induces remodelling of clustered gephyrin in vivo. As a proof-of-principle, we finally tested whether rMS affects gephyrin scaffolds in the intact animal. In this series of experiments, $10-\mathrm{Hz}$ rTMS of anaesthetized 3-month-old male wild-type mice was employed (Fig. 8). The experimental conditions were adapted to our slice culture experiments: (1) orientation of the coil was matched with respect to stimulation of the hippocampus in horizontal slices, that is, portions of the ventral hippocampus used for our slice preparations. (2) The same $10-\mathrm{Hz}$ protocol was employed (at $90 \%$ motor threshold; $~ 60 \%$ of maximum stimulator output, $\mathrm{MSO}^{30}$ ). (3) Animals were killed $2 \mathrm{~h}$ after stimulation and horizontal brain slices containing the hippocampus were stained for gephyrin (Fig. 8a-c). (4) Gephyrin cluster sizes and cluster numbers were assessed in the CA1 stratum pyramidale and stratum radiatum (Fig. 8b,c). Indeed, a significant reduction in gephyrin cluster sizes and numbers was observed in the stratum radiatum but not the stratum pyramidale $2 \mathrm{~h}$ after stimulation (Fig. 8d,e). Together, these experiments demonstrate that $10-\mathrm{Hz}$ rMS is a potent tool to induce the remodelling of inhibitory postsynapses, that is, gephyrin scaffolds in vitro and in vivo.

\section{Discussion}

Alterations in E/I balance and disturbed cortical homeostasis ${ }^{31-33}$ have been suggested to cause behavioural and cognitive dysfunction in many brain diseases, such as schizophrenia ${ }^{11,12}$, autism $^{10,13}$ and panic disorders ${ }^{34}$. In these disease contexts, the diagnostic and therapeutic potentials of non-invasive brain stimulation techniques have been extensively studied ${ }^{1}$. Still, the cellular and molecular mechanisms of rTMS-mediated neural plasticity and hence rTMS-based therapies remain incompletely understood $^{2-4}$. While it has been proposed that LTP/LTD-like plasticity of excitatory synapses underlies rTMS effects on cortical excitability $^{35}$, less attention has been dedicated to rTMS-induced changes in inhibitory neurotransmission ${ }^{36-38}$. The present study demonstrates that $10-\mathrm{Hz}$ rMS induces structural and functional plasticity of inhibitory synapses. These changes depend on the activation of VGSCs, L-VGCCs and NMDARs and are not observed when calcineurin protein phosphatases are pharmacologically blocked. Since dendritic inhibition appeared to 
a
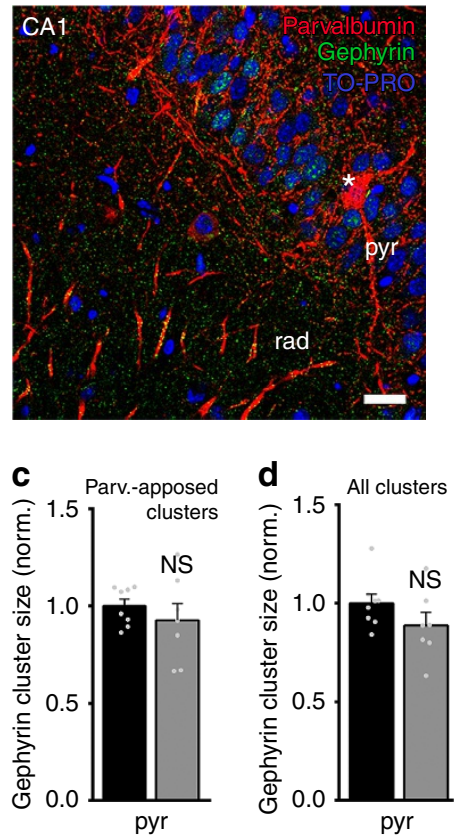

b

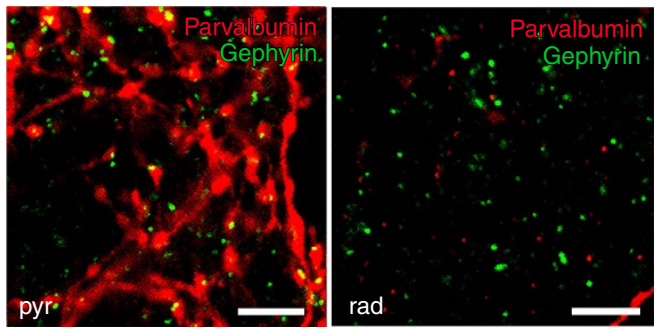

$\mathbf{f}$
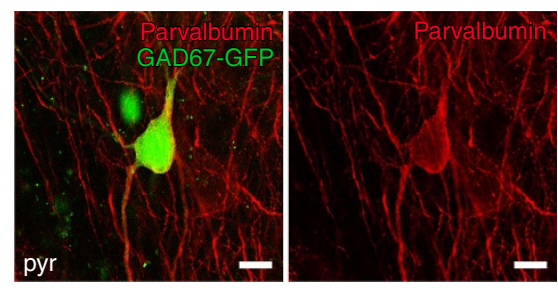

g

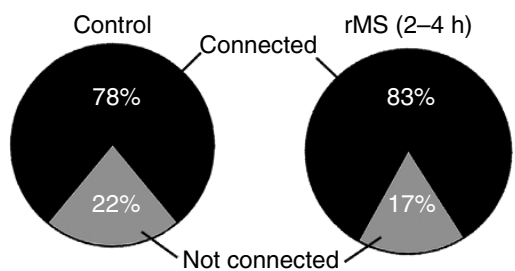

h

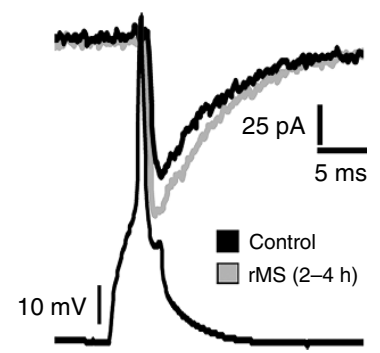

i

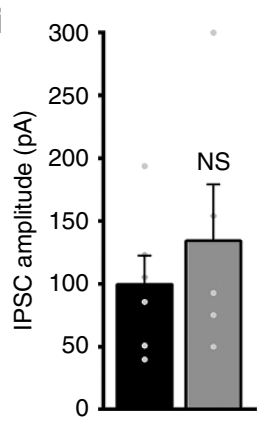

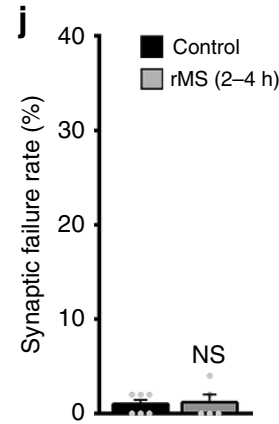

Figure 6 | rMS does not affect somatic inhibition. (a,b) Slice cultures stained for gephyrin (green) and parvalbumin (red). Note the parvalbumin-positive interneuron (asterisk in $\mathbf{a}$ ) in pyr. The axons of these neurons arborize mainly within the pyramidal cell layer (b; rad). The size of gephyrin clusters associated with parvalbumin-positive presynaptic boutons was assessed in pyr. Blue, TO-PRO nuclear stain. Scale bar in a, $20 \mu \mathrm{m}$, in $\mathbf{b}, 4 \mu \mathrm{m}$. (c) No significant difference in parvalbumin-associated gephyrin cluster sizes is observed in pyr between stimulated and non-stimulated slice cultures $3 \mathrm{~h}$ after rMS (control, $n=8$ cultures; rMS, $n=7$ cultures; averaged data from three visual fields per culture; Mann-Whitney test). (d,e) The mean size and number of all gephyrin clusters are not significantly different in pyr. In the rad a significant reduction in gephyrin cluster sizes and numbers is observed after stimulation in the same set of cultures (control, $n=8$ cultures; $r M S, n=7$ cultures; averaged data from three visual fields per culture; Mann-Whitney test). (f) Paired recordings of parvalbumin-positive interneurons and CA1 pyramidal neurons were carried out in slice cultures prepared from GAD67-GFP mice. Parvalbumin-positive interneurons in pyr can be readily identified by the GFP signal in these preparations. Scale bar, $10 \mu \mathrm{m}$. (g-j) No major changes in inhibitory synaptic strength, efficacy and connectivity are observed in these experiments (control; $n=6$ connected pairs in four cultures, one connected pair excluded from analysis since series resistance changed significantly during recordings and reached $\geq 30 \mathrm{M} \Omega$; seven of nine probed pairs connected; rMS, $n=5$ connected pairs in three cultures; five of six probed pairs connected; Mann-Whitney test). Individual data points are indicated by grey dots. Values represent mean \pm s.e.m. ( ${ }^{\star \star} P<0.01 ; n s$, not significant differences).

be mainly affected in our experiments, we conclude that $10-\mathrm{Hz}$ rMS acts through the $\mathrm{Ca}^{2+}$-dependent remodelling of specific, that is, dendritic inhibitory synapses of principal neurons. These results disclose a mechanism how rTMS could modulate E/I balance and connectivity in neuronal networks.
We regard it as one of the major findings of our study that rMS is able to induce the remodelling of gephyrin scaffolds. Gephyrin is thought to form a hexagonal lattice beneath the postsynaptic membrane ${ }^{39-41}$ and to determine synaptic efficacy by immobilizing $\mathrm{GABA}_{\mathrm{A}} \mathrm{Rs}$ and glycine receptors at synaptic 


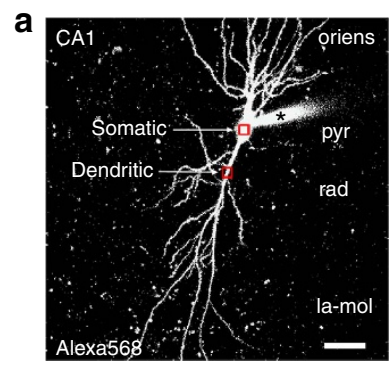

b
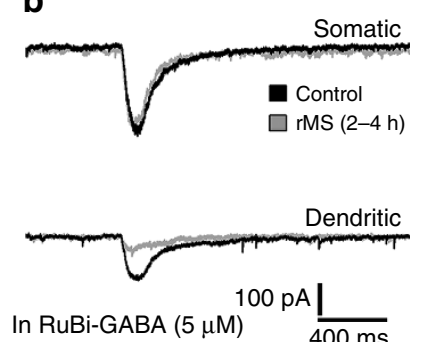

C
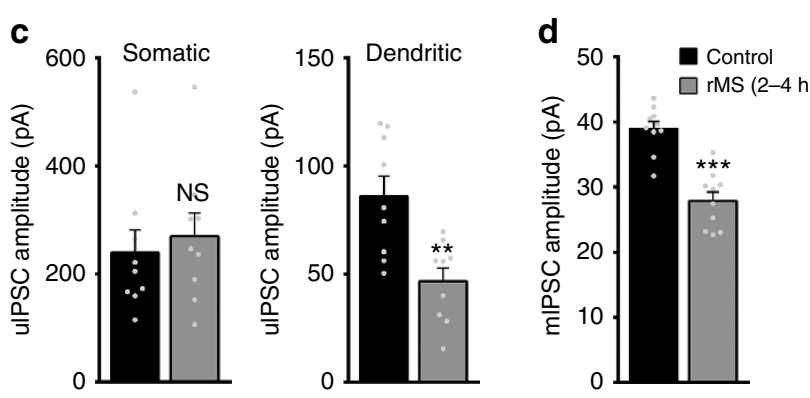

Figure 7 | Flash photolysis of caged GABA discloses that rMS affects predominantly dendritic inhibition. (a) Flash photolysis of RuBi-GABA was employed to separately assess dendritic and somatic inhibition in the same set of patched CA1 pyramidal neurons (filled with Alexa568 before recordings; la-mol). Scale bar, $30 \mu \mathrm{m}$. (b) Representative averaged traces (five consecutive laser-light pulses at $0.1 \mathrm{~Hz}$ in each region) showing RuBiGABA-uncaging-evoked somatic and dendritic responses recorded from non-stimulated control cultures and cultures between 2 and $4 \mathrm{~h}$ after rMS. $(\mathbf{c}, \mathbf{d})$ Group data of uncaging-evoked inhibitory postsynaptic current (uIPSC) amplitudes ( $n=9$ neurons per group; five cultures each; MannWhitney test). In the same set of cultures mIPSC recordings confirmed a significant reduction in the mean mIPSC amplitude of CA1 pyramidal neurons (note: internal solution contained higher $\left[\mathrm{Cl}^{-}\right]$than in Fig. 1h; control, $n=10$ neurons; rMS, $n=10$ neurons from four cultures each; Mann-Whitney test). Individual data points are indicated by grey dots. Values represent mean \pm s.e.m. $\left({ }^{\star \star} P<0.01\right.$; ${ }^{\star \star \star} P<0.001$; ns, not significant differences).

sites $^{42-48}$. Both the receptors and gephyrin are continuously exchanged because of dissociation from and re-association to postsynaptic sites ${ }^{49,50}$. The size and properties of gephyrin clusters are regulated by neuronal activity ${ }^{17,19}$ and phosphorylation ${ }^{39,51}$, and depend on interactions with the cytoskeleton ${ }^{52}$. As discussed elsewhere ${ }^{14}$, synaptic signalling pathways triggered by $\mathrm{Ca}^{2+}$ influx ${ }^{53}$ are considered to regulate inhibitory postsynaptic scaffold dynamics and thereby to control $\mathrm{GABA}_{\mathrm{A}} \mathrm{R}$ recruitment and internalization rates. The results of the present study are in line with this suggestion, since inhibition of NMDARs and L-VGCCs abolished the rMS-induced reduction in gephyrin cluster size and stability in the stratum radiatum. While our MDL-28170 experiments did not support a role of calpainmediated gephyrin degradation ${ }^{18}$, we were able to provide evidence that calcineurin-regulated phosphorylation/dephosphorylation reactions are involved in rMS-induced remodelling of inhibitory postsynapses (see also refs $39,53,54$ ). Hence, it is likely that rMS acts on inhibitory neurotransmission by modulating the $\mathrm{Ca}^{2+} /$ calcineurin-dependent gephyrin oligomerization/dissociation rather than gephyrin synthesis/degradation; a suggestion that is also supported by our qPCR and western blot results on unaltered gephyrin mRNA and protein levels $3 \mathrm{~h}$ after rMS in vitro. Whether the rMS-induced destabilization of gephyrin scaffolds, the reduction in gephyrin cluster sizes and gephyrin cluster numbers resemble distinct mechanisms that share overlapping signalling
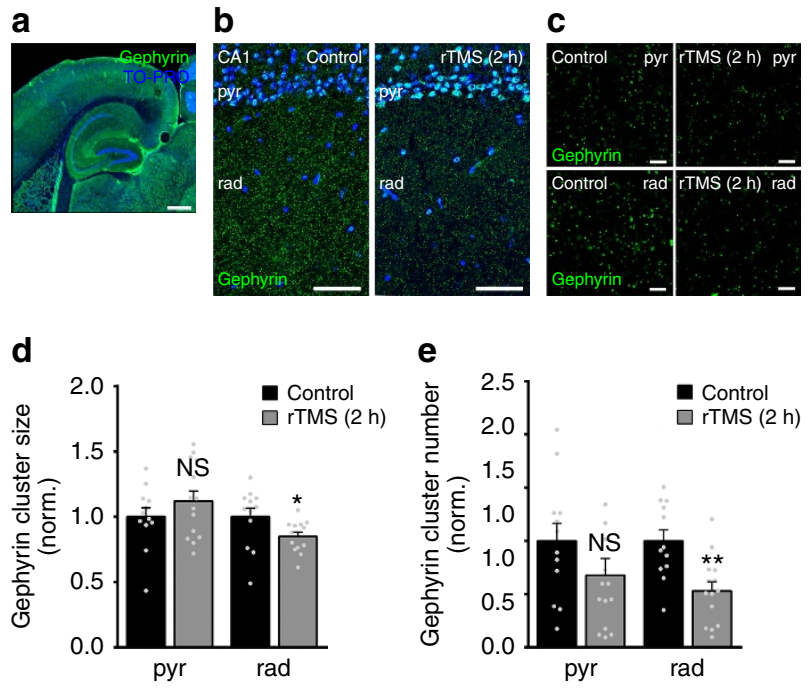

Figure 8 | TMS induces changes in gephyrin clusters in vivo.

(a-c) Examples of horizontal brain slices containing the hippocampus stained for gephyrin from non-stimulated anaesthetized 3-month-old male mice and $2 \mathrm{~h}$ after $10-\mathrm{Hz}$ rTMS. Experimental conditions were adapted to the in vitro experiments. An increase in gephyrin immunostaining co-localizing with TO-PRO nuclear stain (blue) was noted. Scale bar in $\mathbf{a}$, $300 \mu \mathrm{m}$, in b, $50 \mu \mathrm{m}$, in $\mathbf{c}, 4 \mu \mathrm{m}$. (d,e) A significant reduction in gephyrin cluster sizes and numbers was observed in rad but not in pyr $2 \mathrm{~h}$ after stimulation (control, $n=12$ hippocampi from six animals; rTMS, $n=14$ hippocampi from seven animals; Mann-Whitney test). Individual data points are indicated by grey dots. Values represent mean \pm s.e.m. $\left({ }^{\star} P<0.05 ;{ }^{\star \star} P<0.01 ;\right.$ ns, not significant differences).

pathways, or different stages of the same process needs to be determined (c.f., differential effect of NMDAR inhibition on rMSinduced gephyrin cluster size/stability and numbers; Table 1). Regardless of these considerations, our study provides robust experimental evidence that rMS is a suitable tool to induce the remodelling of gephyrin scaffolds (both in vitro and in vivo). In light of the fact that rare exonic deletions implicate gephyrin in risk for autism, schizophrenia and seizures ${ }^{55}$, we propose that a better understanding of rMS-mediated changes in gephyrin-dependent inhibitory synaptic plasticity (under physiological and pathological conditions) may provide the perspective to transfer basic science knowledge into clinical practice and eventually devise new diagnostic and therapeutic strategies using non-invasive brain stimulation techniques.

Using immunostainings for gephyrin, GABA-uncaging experiments and paired recordings, we demonstrate that rMS changes the strength, efficacy and connectivity of dendritic inhibition, while having no major impact on somatic inhibition. The precise mechanisms through which rMS exerts its effects predominantly on dendritic (but not somatic) inhibition warrant further investigation. For example, differential effects of the induced electric field on somatic versus dendritic compartments, on specific subtypes of interneurons or glial cells are possible. Alternatively, intracellular calcium stores and the distribution of distinct channels, receptor (subunits) and enzymes may play a role. It is also possible that inhibitory synapses in the soma are less susceptible to $\mathrm{Ca}^{2+}$-mediated remodelling. Thus, our $10-\mathrm{Hz}$ rMS protocol may not induce somatic $\mathrm{Ca}^{2+}$ levels high enough to trigger plastic changes of inhibitory postsynapses in this compartment. The requirement of both NMDAR and L-VGCC activity during stimulation indicates that a synergistic activation of these $\mathrm{Ca}^{2+}$ entry sites is required for the local remodelling of 
inhibitory postsynapses to occur after rMS. Since pyramidal cells of the cortex do not carry many asymmetric, that is, NMDARcontaining excitatory synapses on their soma ${ }^{56}$, this synergy may simply not occur at the soma.

Although the functional consequences of rMS-induced changes in inhibition for network function and behaviour ${ }^{57-59}$ remain unknown, it is interesting to hypothesize that rTMS may assert its beneficial effects seen in the context of neuropsychiatric diseases by modulating specific molecular and functional aspects of inhibition in cortical networks, for example, dendritic inhibition. This suggestion is supported by recent experimental evidence that indicates that the recruitment of disinhibitory microcircuits plays an important role in cortical plasticity ${ }^{7}$. Hence, it is conceivable that rMS-induced disinhibition could prime cortical networks for the expression of subsequent (experience-dependent) plasticity. However, at this point we have to concede that we do not know enough about the temporal sequence and molecular interdependency of rMS-induced disinhibition and other forms of plasticity, except that both rMS-induced LTP of excitatory synapses $^{5,6}$ and LTD of inhibition (this study) require the activation of VGSCs, L-VGCCs and NMDARs during stimulation.

It will now be important to test whether the outcome of rMS on synaptic plasticity depends on the architecture and state of the stimulated network or the specific stimulation protocol employed. Differential effects of rMS on specific inhibitory (and excitatory) synapses might be observed when stimulus intensities, frequencies, total number of applied magnetic pulses and the orientation of the stimulated tissue within the electromagnetic field are modified $^{60}$, or under conditions in which E/I balance is pharmacologically or genetically altered (for example, to mimic specific pathological brain conditions; see also ref. 30 , who describe differential outcomes of rTMS in anaesthetized and awake animals). Nevertheless, by carefully adapting our stimulation parameters to the in vivo situation we could verify that rMS-induced changes in gephyrin scaffolds are also seen in the intact animal situation. We are confident that future in vitro and in vivo studies of the hippocampus and other brain regions, possibly in combination with computational approaches, which will help to explore the enormous parameter space, will provide novel insights on the dose-, orientation- and state dependency of rMS-mediated neural plasticity and its outcome on E/I balance under physiological and pathological conditions. These studies may support the transfer of basic science knowledge on neural plasticity into clinical practice and could thereby also help addressing some of the important questions regarding inter- and intraindividual variabilities of rTMS effects in human subjects ${ }^{2,61}$.

\footnotetext{
Methods

Ethics statement. Mice were maintained in a 12-h light/dark cycle with food and water available ad libitum. Every effort was made to minimize distress and pain of animals. All experimental procedures were performed according to the German animal welfare legislation and approved by the appropriate animal welfare committee and the animal welfare officer of Goethe-University Frankfurt, Faculty of Medicine.
}

Preparation of slice cultures. Entorhinohippocampal slice cultures were prepared on postnatal days 4-5 from C57BL/6J, Thy1-GFP/gephyrin (heterozygous for GFPGephyrin) ${ }^{17}, G A D 65-G F P^{24}$ and GAD67-GFP ${ }^{28}$ (heterozygous for GFP; obtained from Jackson Laboratories, USA) mice of either $\operatorname{sex}^{17}$. Cultivation medium contained 50\% (v/v) MEM, 25\% (v/v) basal medium eagle, 25\% (v/v) heatinactivated normal horse serum (NHS), $25 \mathrm{mM}$ HEPES buffer solution, $0.15 \%$ $(\mathrm{w} / \mathrm{v})$ bicarbonate, $0.65 \%(\mathrm{w} / \mathrm{v})$ glucose, $0.1 \mathrm{mg} \mathrm{ml}^{-1}$ streptomycin, $100 \mathrm{U} \mathrm{ml}^{-1}$ penicillin and $2 \mathrm{mM}$ glutamax. The $\mathrm{pH}$ was adjusted to 7.3 and the medium was replaced three times per week. All slice cultures were allowed to mature for at least 18 days in humidified atmosphere with $5 \% \mathrm{CO}_{2}$ at $35^{\circ} \mathrm{C}$. Cultures prepared from at least three independent litters were used in each biological experiment.
rMS in vitro. Slice cultures ( $\geq 18$ days in vitro) were transferred to a non-tem perature-controlled chamber, that is, a $30-\mathrm{mm}$ Petri dish containing standard extracellular solution $(129 \mathrm{mM} \mathrm{NaCl}, 4 \mathrm{mM} \mathrm{KCl}, 1 \mathrm{mM} \mathrm{MgCl}, 2 \mathrm{mM} \mathrm{CaCl}$, $4.2 \mathrm{mM}$ glucose, $10 \mathrm{mM}$ HEPES, $0.1 \mathrm{mg} \mathrm{ml}^{-1}$ streptomycin, $100 \mathrm{U} \mathrm{ml}^{-1}$ penicillin, $\mathrm{pH} 7.4$ with $\mathrm{KOH}$; preheated to $35^{\circ} \mathrm{C}$; osmolarity adjusted with sucrose to match cultivation medium). Cultures were stimulated in the absence of any wires and electrodes using a Magstim Rapid stimulator with a biphasic current waveform, using two booster modules (Magstim Company, UK) and connected to a standard 70-mm outer wing diameter double, that is, figure-of-eight coil (Magstim Company). Cultures were positioned $1 \mathrm{~cm}$ under the centre of the coil (that is, junction of the two wings) and stimulated with a protocol consisting of nine trains of 100 pulses each at $10 \mathrm{~Hz}$ with an intertrain interval of $30 \mathrm{~s}$ (at $50 \%$ of MSO). Orientation of cultures was such that the induced electric field within the tissue was approximately parallel to the dendritic tree of CA1 pyramidal neurons. In some experiments, TTX $(2 \mu \mathrm{M})$, AP5 $(50 \mu \mathrm{M})$ or Nifedipin $(20 \mu \mathrm{M})$ were used to block VGSCs, NMDARs or L-VGCCs during stimulation, respectively. Drugs were immediately washed out after stimulation. Cultures were kept in the incubator for at least $2 \mathrm{~h}$ after stimulation before experimental assessment. Cyclosporin $\mathrm{A}(2 \mu \mathrm{M})$ or MDL-28170 $(50 \mu \mathrm{M})$ were used to block the $\mathrm{Ca}^{2+}$-dependent phosphatase calcineurin or the $\mathrm{Ca}^{2+}$-dependent protease calpain, respectively. Age- and timematched control cultures were not stimulated, but otherwise treated identical to stimulated cultures.

Modelling the electric field in vitro. The electric field induced by rMS in vitro was described based on a magnetic dipole model of the Magstim 70-mm figure-ofeight coil $^{62}$. A finite element method model was created with the dimensions of the bath and tissue adapted to the in vitro setting. The coil was positioned $1 \mathrm{~cm}$ above the tissue. Electrical conductivities were $0.3 \mathrm{~S} \mathrm{~m}^{-1}$ for the tissue and $1.4 \mathrm{~S} \mathrm{~m}^{-1}$ for

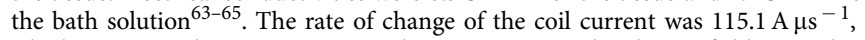
which corresponds to $50 \%$ MSO in the experiments. The electric field was solved using a quasistatic approximation ${ }^{66}$ with the SimNibs toolbox ${ }^{67}$. The computed electric field $\left(27.3 \pm 3.3 \mathrm{~V} \mathrm{~m}^{-1}\right)^{68}$ in the tissue was nearly perfectly aligned along the coil axis with a mean absolute angular deviation of $4.3^{\circ}$. The effects of slight changes in coil-to-slice culture distance were estimated by comparing the induced electric field strengths for distances of $1,1.1$ and $1.2 \mathrm{~cm}$. Under these conditions, differences of $<2.5 \mathrm{~V} \mathrm{~m}^{-1}$ were determined.

rTMS of anaesthetized mice. rTMS was carried out in 3-month-old male urethane-anaesthetized $C 57 B L / 6 J$ mice $\left(1.25 \mathrm{~g} \mathrm{~kg}^{-1}\right.$, intraperitoneal; $0.125 \mathrm{~g} \mathrm{~kg}^{-1}$, subcutaneous). The head was placed under the centre of the coil and the orientation of the coil was optimized to mirror the in vitro setting, in which the hippocampus taken from horizontal brain slices is used for cultivation and subsequent rMS. Brain-to-coil distance was kept minimal while assuring contact-free stimulation. The motor threshold was determined before each experiment using single pulses at $0.2 \mathrm{~Hz}$ with increasing stimulator intensity (5\% MSO steps, starting from $40 \%$ MSO). Repetitive stimulation was performed at $90 \%$ of motor threshold (corresponding to $59 \pm 1 \% \mathrm{MSO}$ ) using the same $10-\mathrm{Hz}$ stimulation protocol described above. Control animals placed near the coil during stimulation were not stimulated but otherwise treated identically to stimulated animals. All animals were transferred back to their cages and held in anaesthesia for $2 \mathrm{~h}$. Deeply anaesthetized mice were rapidly decapitated. Brains were removed and briefly washed in ice-cold PBS before embedding in Tissue-Tek freezing medium (Sakura Finitek). After 3 min in 2 -methylbutan at $-40^{\circ} \mathrm{C}$ brains were stored at $-20^{\circ} \mathrm{C}$.

Immunostaining and imaging. Slice cultures were fixed in a solution of $4 \%(\mathrm{w} / \mathrm{v})$ paraformaldehyde (PFA) in PBS (0.1 M, pH 7.4) and 4\% (w/v) sucrose for $1 \mathrm{~h}$, followed by $2 \%$ PFA and $30 \%$ sucrose in PBS overnight. Cryostat sections $(30 \mu \mathrm{m})$ of fixed slice cultures were prepared and stained with antibodies against gephyrin (Synaptic Systems, clone mAb7a; 1:500), GABA ${ }_{\mathrm{A}} \mathrm{R} \alpha 2$ (Santa Cruz Biotechnology, sc-7350; 1:500), GABA ${ }_{\mathrm{A}} \mathrm{R} \alpha 5$ (Sigma-Aldrich, SAB-2701358; 1:500) and/or parvalbumin (Swant, PV-28; 1:200) following a modified protocol previously described ${ }^{69}$ Briefly, the sections were incubated for $1 \mathrm{~h}$ with $10 \%(\mathrm{v} / \mathrm{v})$ normal goat serum (NGS) or NHS in $0.5 \%(\mathrm{v} / \mathrm{v})$ Triton X-100 containing PBS to reduce unspecific staining and subsequently incubated for $48 \mathrm{~h}$ at $4{ }^{\circ} \mathrm{C}$ with the respective primary antibodies (in PBS with 10\% NGS or NHS and $0.1 \%$ Triton X-100). Sections were washed and incubated for $3 \mathrm{~h}$ with appropriate Alexa 488 or 568 -labelled secondary antibodies (Invitrogen; 1:1,000, in PBS with 10\% NGS or NHS, $0.1 \%$ Triton X-100). TO-PRO (Invitrogen) nuclear stain was used to visualize cytoarchitecture (1:5,000; in PBS for $10 \mathrm{~min})$. The sections were washed, transferred on glass slides and mounted for visualization with anti-fading mounting medium.

Horizontal cryostat sections $(35 \mu \mathrm{m})$ containing the ventral hippocampus were prepared from whole-mouse brains and immediately mounted on glass slides. Sections were fixed with $4 \% \mathrm{PFA} / 4 \%$ sucrose for $15 \mathrm{~min}$. After washing with PBS, the slices were incubated for $1 \mathrm{~h}$ with $10 \%$ NGS in $0.5 \%$ Triton X-100 containing PBS to reduce unspecific staining and subsequently incubated overnight at $4{ }^{\circ} \mathrm{C}$ with mouse anti-gephyrin antibody (Synaptic Systems, clone mAb7a; 1:500, in PBS with $10 \%$ NGS and $0.1 \%$ Triton X-100). Sections were washed and incubated for $2 \mathrm{~h}$ with Alexa488-labelled goat anti-mouse antibody (Invitrogen; 1:500, 10\% NGS, $0.1 \%$ Triton X-100). TO-PRO (Invitrogen) nuclear stain was used to visualize 
cytoarchitecture (1:5,000; in PBS for $10 \mathrm{~min})$. Sections were washed again, transferred on glass slides and mounted for visualization with anti-fading mounting medium.

A Nikon Eclipse C1si laser-scanning microscope with a $\times 4$ objective lens (numeric aperture (NA) 0.2 , Nikon), a $\times 40$ objective lens (NA 1.3, Nikon) and a $\times 60$ oil-immersion objective lens (NA 1.4, Nikon) was used for confocal microscopy. Three visual fields per region of interest were imaged in each culture at high magnification. Detector gain and amplifier were set to obtain pixel intensities within a linear range.

\section{Fluorescence recovery after photobleaching. FRAP experiments were per-} formed with a Zeiss LSM Exciter confocal microscope at $35^{\circ} \mathrm{C}$ (bath solution contained $126 \mathrm{mM} \mathrm{NaCl}, 2.5 \mathrm{mM} \mathrm{KCl}, 26 \mathrm{mM} \mathrm{NaHCO}_{3}, 1.25 \mathrm{mM} \mathrm{NaH}_{2} \mathrm{PO}_{4}$, $2 \mathrm{mM} \mathrm{CaCl}_{2}, 2 \mathrm{mM} \mathrm{MgCl}_{2}$ and $10 \mathrm{mM}$ glucose $)^{17,69}$. Individual GFP-gephyrin clusters in the stratum radiatum of area CA1 were imaged with a $\times 40$ water immersion objective lens $(0.8 \mathrm{NA}$; Zeiss $)$ and $\times 2$ scan zoom. The pinhole diameter was set at 1 Airy Unit, and image stacks (seven images) were taken at an ideal Nyquest rate. Imaging parameters were optimized to minimize bleaching by the imaging procedure. Following a 15 -min baseline registration $(\Delta t=5 \mathrm{~min})$, selected GFP-gephyrin clusters in the middle plane of the stack were photobleached $(<5 \%$ of initial fluorescence) using the bleaching function of the Zeiss Zen Software (Acousto Optic Tunable Filter, AOTF-controlled Argon laser 488 nm; 100\% transmission; 100 bleach iterations) and FRAP was followed for $80 \mathrm{~min}$ ( $\Delta t=5 \mathrm{~min}$ ). Our earlier work revealed that in the CA1 stratum radiatum of entorhinohippocampal slice cultures $\sim 92 \%$ of the GFP-gephyrin clusters are synaptically localized, as revealed by co-immunolabelling for the presynaptic vesicular inhibitory amino-acid transporter ${ }^{17}$.

Western blotting. Isolated tissue containing the CA1 region was separated on $8 \%$ (w/v) SDS-polyacrylamide gels $(30 \mu \mathrm{g}$ protein per lane), transferred to nitrocellulose (Protran Whatman) and probed with anti-gephyrin (Synaptic Systems, 14711; 1:3,000) and anti-GAPDH (Calbiochem, CB1001; 1:10,000), followed by incubation with appropriate secondary antibodies (LI-COR, IRDye800 or IRDye680-conjugated antibodies; 1:10,000). Bound antibody was visualized using the Odyssey Infrared Imaging System (LICOR).

RNA extraction and $q P C R$. RNA from tissue containing the CA1 region was isolated using the RNeasy MicroPlus Kit (Qiagen). RNA integrity numbers $(9.7 \pm 0.04)$ were determined using the Agilent 2100 Bioanalyzer system and Agilent RNA 6000 Pico Kit (Agilent Technologies, Germany). The High-Capacity cDNA Reverse Transcription Kit (Applied Biosystems, USA) was used to transcribe purified RNA into cDNA (all kits and assays used according to the manufacturers' instructions). The cDNA was amplified with the TaqManPreAmp Master Mix Kit (Applied Biosystems) using $5 \mu \mathrm{l}$ PreAmp Master Mix (Applied Biosystems) $+2.5 \mu \mathrm{l}$ cDNA $+2.5 \mu$ l Assay Mix (TaqMan Gene Expression (TM)-Assay (Gephyrin: Mm00556895_m1; GAPDH: 4352932E) from Applied Biosystems) with a standard amplification protocol $\left(14\right.$ cycles: $95^{\circ} \mathrm{C}$ for $15 \mathrm{~s} ; 60^{\circ} \mathrm{C}$ for $\left.4 \mathrm{~min}\right)$. Amplified cDNAs were diluted 1:20 in ultrapure water and subjected to qPCR (StepOnePlus, Applied Biosystems) using a standard amplification programme $\left(1 \mathrm{cycle}\right.$ of $50^{\circ} \mathrm{C}$ for $2 \mathrm{~min}, 1 \mathrm{cycle}$ of $95^{\circ} \mathrm{C}$ for $10 \mathrm{~min}, 40$ cycles of $95^{\circ} \mathrm{C}$ for $15 \mathrm{~s}$ and $60^{\circ} \mathrm{C}$ for $60 \mathrm{~s}$; cutoff at 36 cycles; averaged $C_{\mathrm{t}}$ value was: $22.9 \pm 0.2$ cycles).

Whole-cell patch-clamp recordings. Whole-cell voltage-clamp recordings were carried out at $35^{\circ} \mathrm{C}$ (two to five neurons per culture) ${ }^{5}$. The bath solution contained $126 \mathrm{mM} \mathrm{NaCl}, 2.5 \mathrm{mM} \mathrm{KCl}, 26 \mathrm{mM} \mathrm{NaHCO}_{3}, 1.25 \mathrm{mM} \mathrm{NaH}_{2} \mathrm{PO}_{4}, 2 \mathrm{mM} \mathrm{CaCl}_{2}$, $2 \mathrm{mM} \mathrm{MgCl}_{2}$ and $10 \mathrm{mM}$ glucose. For mIPSC recordings, patch pipettes contained $40 \mathrm{mM} \mathrm{CsCl}, 90 \mathrm{mM}$ K-gluconate, $1.8 \mathrm{mM} \mathrm{NaCl}, 1.7 \mathrm{mM} \mathrm{MgCl}_{2}, 3.5 \mathrm{mM} \mathrm{KCl}$, $0.05 \mathrm{mM}$ EGTA, $2 \mathrm{mM}$ ATP-Mg, $0.4 \mathrm{mM}$ GTP-Na $2,10 \mathrm{mM}$ PO-Creatine, $10 \mathrm{mM}$ HEPES ( $\mathrm{pH}=7.25$ with $\mathrm{KOH}, 290 \mathrm{mOsm}$ with sucrose), having a tip resistance of 4-6 M $\Omega$. Alexa488 or Alexa568 (both $10 \mu \mathrm{M}$ ) was added to the internal solution in some experiments to visualize neuronal morphology before recordings. Neurons were recorded at holding potential $-80 \mathrm{mV}$ in the presence of $0.5 \mu \mathrm{M}$ TTX, $10 \mu \mathrm{M}$ AP5 and $10 \mu \mathrm{M}$ CNQX. Series resistance was monitored in 2-min intervals, and recordings were discarded if the series resistance and leak current changed significantly and/or reached $\geq 30 \mathrm{M} \Omega$ or $\geq 350 \mathrm{pA}$, respectively. All electrophysiological recordings were performed from stimulated and nonstimulated slice cultures between 2 and $4 \mathrm{~h}$ after stimulation in a pseudorandomized manner to avoid acquisition bias.

Tonic $\mathbf{G A B A}_{\mathbf{A}}$ receptor conductance. Patch pipettes contained $125 \mathrm{mM} \mathrm{CsCl}$, $5 \mathrm{mM} \mathrm{NaCl}, 2 \mathrm{mM} \mathrm{MgCl} 2,2 \mathrm{mM} \mathrm{Mg}$-ATP, $0.5 \mathrm{mM} \mathrm{Na}_{2}$-GTP, $0.1 \mathrm{mM}$ EGTA and $10 \mathrm{mM}$ HEPES ( $\mathrm{pH}=7.33$ with $\mathrm{CsOH} ; 274 \mathrm{mOsm}$ with sucrose). $\mathrm{GABA}_{\mathrm{A}} \mathrm{R}$ currents were isolated using $0.5 \mu \mathrm{M}$ TTX, $10 \mu \mathrm{M}$ AP5 and $10 \mu \mathrm{M}$ CNQX and in the bath solution, which enabled us to also detect mIPSC in the same set of recordings. The amplitude of tonic $\mathrm{GABA}_{\mathrm{A}} \mathrm{R}$ current was determined by perfusing GABA $(5 \mu \mathrm{M})$, followed by bicuculline-methiodide $(50 \mu \mathrm{M})$ containing bath solution.

Paired recordings. Simultaneous whole-cell patch-clamp recordings of neurons were carried out as described previously ${ }^{5,29}$. Slice cultures prepared from GAD65$G F P^{24}$ or GAD67-GFP mice were used to readily identify interneurons projecting their axons on dendrites or the soma of CA1 pyramidal neurons, respectively.
Internal solution for presynaptic recordings of GFP-expressing interneurons contained $126 \mathrm{mM}$ K-gluconate, $4 \mathrm{mM} \mathrm{KCl}, 4 \mathrm{mM}$ ATP-Mg, $0.3 \mathrm{mM} \mathrm{Na} \mathrm{m}_{2}$ GTP, $10 \mathrm{mM}$ PO-creatine, $10 \mathrm{mM}$ HEPES and $0.1 \%$ biocytin $(\mathrm{pH}=7.25$ with $\mathrm{KOH}$, 290 mOsm with sucrose). CA1 pyramidal neurons in GAD67-GFP slices were recorded with low chloride solution (c.f., mIPSC recordings) and in GAD65-GFP preparations with high chloride solution (c.f., tonic inhibition). Action potentials were generated by $3-\mathrm{ms}$ square current pulses $(1 \mathrm{nA}$ ) elicited at $0.1 \mathrm{~Hz}$ (up to 50 pulses) while recording inhibitory postsynaptic currents from CA1 pyramidal neurons (recordings performed in the presence of AP5 and CNQX, both $10 \mu \mathrm{M}$; since these recordings were performed in the absence of TTX, we were also able to assess rMS-induced changes in spontaneous IPSCs). Paired-pulse kinetics of IPSCs were determined by inducing two presynaptic action potentials with increasing interpulse interval $(20-200 \mathrm{~ms}, \Delta t=20 \mathrm{~ms}$ at $0.1 \mathrm{~Hz}$; at least four repetitions). For short-term plasticity, five action potentials were applied at $20 \mathrm{~Hz}$ (intersweep interval: $10 \mathrm{~s} ; 30$ repetitions).

Flash photolysis of caged GABA. Alexa568 $(10 \mu \mathrm{M})$ was added to the internal solution (125 mM CsCl, $5 \mathrm{mM} \mathrm{NaCl}, 2 \mathrm{mM} \mathrm{MgCl} 2,2 \mathrm{mM} \mathrm{Mg-ATP}, 0.5 \mathrm{mM}$ $\mathrm{Na}_{2}$-GTP, $0.1 \mathrm{mM}$ EGTA and $10 \mathrm{mM}$ HEPES; $\mathrm{pH}=7.33$ with $\mathrm{CsOH} ; 274 \mathrm{mOsm}$ with sucrose) to visualize cellular morphology before recordings. For local stimulation of $\mathrm{GABA}_{\mathrm{A}} \mathrm{Rs}, \mathrm{CA} 1$ pyramidal neurons were recorded in presence of RuBiGABA $(5 \mu \mathrm{M}$; Tocris Bioscience; in $0.5 \mu \mathrm{M}$ TTX, $10 \mu \mathrm{M}$ AP5 and $10 \mu \mathrm{M}$ CNQX containing bath solution). The laser beam was focused on the maximal cross-sectional area of the soma or the principal apical dendrite within the stratum radiatum at a distance of $\sim 50 \mu \mathrm{m}$ from the soma (Zeiss LSM Exciter confocal microscope equipped with a Zeiss $\times 40$ water immersion objective lens; NA 0.8). A region of interest $\left(30 \times 30\right.$ pixels; $\left.\sim 75 \mu \mathrm{m}^{2}\right)$ was selected (soma versus dendrite), and photolysis was performed using the bleaching function of the Zeiss Zen software (AOTF-controlled Argon laser $488 \mathrm{~nm} ; 100 \%$ transmission; single bleach iteration, $<1 \mathrm{~ms}$ duration; five times at $0.1 \mathrm{~Hz}$ per region), while recording inward current responses from the soma of CA1 pyramidal neurons in whole-cell voltage clamp configuration.

Quantification and statistics. Analysis was performed by investigators blind to experimental conditions. Electrophysiological data were analysed using pClamp 10.2 (Axon Instruments) and MiniAnalysis (Synaptosoft) software. Overall, 150 350 mIPSC or sIPSC events were analysed per recorded neuron. Tonic inhibition was analysed by fitting Gaussian distributions to all point histograms for 10-s epochs of the recordings before and after the application of GABA and after the application of bicuculline-methiodide. Owing to the presence of mIPSCs, only the positive sites of the histograms were fitted under baseline conditions and after GABA application ${ }^{70}$. The differences between the means of the Gaussian fits were calculated. Small negative shifts were set to zero, as they are likely to result from variances within noise. Paired recordings and caged-GABA experiments were manually assessed. Network connectivity was estimated by calculating the ratio between connected pairs and the total number of probed pairs. The percentage of action potentials not successfully evoking postsynaptic current responses was determined (synaptic failure rate), as well as the mean amplitude of all successfully evoked postsynaptic responses. Amplitudes of postsynaptic responses were normalized to the first pulse in averaged traces of paired-pulse and short-term plasticity recordings. Individual data points were plotted and linear regression fits were performed using the GraphPad Prism curve fitting toolbox. The mean amplitude of consecutive GABA-uncaging responses was determined in each region (somatic versus dendritic) for each recorded neuron.

Sizes and numbers of immunolabelled gephyrin, $\mathrm{GABA}_{\mathrm{A}} \mathrm{R} \alpha 2$ and $\mathrm{GABA}_{\mathrm{A}} \mathrm{R} \alpha 5$ clusters were assessed using the ImageJ software package (available from http:// rsb.info.nih.gov/ij) ${ }^{6}$. In gephyrin/parvalbumin double-staining experiments, parvalbumin-apposed gephyrin clusters were analysed in the stratum pyramidale. qPCR-data were analysed as described by Pfaffl ${ }^{71}$ with GAPDH serving as reference gene. The qPCR assay efficiency was calculated with the StepOnePlus software (Applied Biosystems) based on a dilution series of five samples for each assay. Western blots were analysed using the Image Studio Software (LICOR).

FRAP of GFP-gephyrin clusters was analysed using the ImageJ software package ${ }^{17}$. Values (corrected for background and bleaching by the imaging procedure) were normalized to prebleach fluorescence and to the first time point after bleaching. All values were expressed as the percentage of the mean prebleach fluorescence (that is, averaged corrected fluorescence of baseline recordings; $\Delta t=5 \mathrm{~min}$ ) for every analysed cluster (5-12 clusters per culture). FRAP data were fitted using the curve fitting toolbox of GraphPad Prism 6 (GraphPad software, USA) with a bi-exponential equation (two phase association, least squares fit):

$$
f(t)=f(\mathrm{t}=0)+P_{\text {fast }}\left(1-\mathrm{e}^{-t * \mathrm{k}(\text { fast })}\right)+P_{\text {slow }}\left(1-\mathrm{e}^{-t * \mathrm{k}(\text { slow })}\right)
$$

where $P_{\text {fast }}$ denotes the fraction of the fast component with the recovery time constant $k_{\text {(fast) }}$ and $P_{\text {slow }}$ the fraction of the slow component with the recovery time constant $k_{\text {(slow) }}$, respectively. Owing to difficulties to reliably determine the often rather small $P_{\text {fast }}$ values observed in some experiments, statistical evaluations were based on comparisons of the averaged FRAP values sampled between 70 and $80 \mathrm{~min}$. Data from non-stimulated pharmacologically treated and untreated cultures were pooled (c.f., Fig. 3e,f and Table 1). 
Sample sizes were chosen according to initial pilot experiments and prior studies that used similar experimental approaches. Power estimation was performed using $\mathrm{G}^{\star}$ Power 3 (Düsseldorf, Germany). Data were analysed using GraphPad Prism 6 (GraphPad software). Statistical comparisons were made using nonparametric tests, since normal distribution could not be assured: MannWhitney test (to compare two groups) or the Kruskal-Wallis test followed by Dunn's post hoc test, which accounts for multiple testing. $P$ values of less than 0.05 were considered a significant difference. All values represent mean \pm s.e.m. In the figures, ${ }^{\star} P<0.05,{ }^{*} P<0.01$ and ${ }^{* *} P<0.001$; not significant differences are indicated with 'ns'.

Digital illustrations. Confocal image stacks were exported as two-dimensional projections and stored as TIFF files. Figures were prepared using the Photoshop graphics software (Adobe, San Jose, CA, USA). Image brightness and contrast were adjusted.

\section{References}

1. Lefaucheur, J. P. et al. Evidence-based guidelines on the therapeutic use of repetitive transcranial magnetic stimulation (rTMS). Clin. Neurophysiol. 125, 2150-2206 (2014).

2. Rothwell, J. C. Clinical applications of noninvasive electrical stimulation: problems and potential. Clin. EEG Neurosci. 43, 209-214 (2012).

3. Dayan, E., Censor, N., Buch, E. R., Sandrini, M. \& Cohen, L. G. Noninvasive brain stimulation: from physiology to network dynamics and back. Nat. Neurosci. 16, 838-844 (2013).

4. Muller-Dahlhaus, F. \& Vlachos, A. Unraveling the cellular and molecular mechanisms of repetitive magnetic stimulation. Front. Mol. Neurosci. 6, 50 (2013).

5. Vlachos, A. et al. Repetitive magnetic stimulation induces functional and structural plasticity of excitatory postsynapses in mouse organotypic hippocampal slice cultures. J. Neurosci. 32, 17514-17523 (2012).

6. Lenz, M. et al. Repetitive magnetic stimulation induces plasticity of excitatory postsynapses on proximal dendrites of cultured mouse CA1 pyramidal neurons. Brain Struct. Funct. 220, 3323-3337 (2015).

7. Letzkus, J. J. et al. A disinhibitory microcircuit for associative fear learning in the auditory cortex. Nature 480, 331-335 (2011).

8. Pecka, M., Han, Y., Sader, E. \& Mrsic-Flogel, T. D. Experience-dependent specialization of receptive field surround for selective coding of natural scenes. Neuron 84, 457-469 (2014).

9. Lovett-Barron, M. et al. Dendritic inhibition in the hippocampus supports fear learning. Science 343, 857-863 (2014).

10. Rubenstein, J. L. \& Merzenich, M. M. Model of autism: increased ratio of excitation/inhibition in key neural systems. Genes Brain Behav. 2, 255-267 (2003).

11. Yizhar, O. et al. Neocortical excitation/inhibition balance in information processing and social dysfunction. Nature 477, 171-178 (2011).

12. Rowland, L. M. et al. GABA predicts inhibition of frequency-specific oscillations in schizophrenia. J. Neuropsychiatry Clin. Neurosci. 25, 83-87 (2013).

13. Rojas, D. C., Singel, D., Steinmetz, S., Hepburn, S. \& Brown, M. S. Decreased left perisylvian GABA concentration in children with autism and unaffected siblings. NeuroImage 86, 28-34 (2014).

14. Kneussel, M. \& Betz, H. Receptors, gephyrin and gephyrin-associated proteins: novel insights into the assembly of inhibitory postsynaptic membrane specializations. J. Physiol. 525(Pt 1): 1-9 (2000).

15. Papadopoulos, T. \& Soykan, T. The role of collybistin in gephyrin clustering at inhibitory synapses: facts and open questions. Front. Cell Neurosci. 5, 11 (2011).

16. Tyagarajan, S. K. \& Fritschy, J. M. Gephyrin: a master regulator of neuronal function? Nat. Rev. Neurosci. 15, 141-156 (2014).

17. Vlachos, A., Reddy-Alla, S., Papadopoulos, T., Deller, T. \& Betz, H. Homeostatic regulation of gephyrin scaffolds and synaptic strength at mature hippocampal GABAergic postsynapses. Cereb. Cortex 23, 2700-2711 (2013).

18. Tyagarajan, S. K. et al. Extracellular signal-regulated kinase and glycogen synthase kinase 3beta regulate gephyrin postsynaptic aggregation and GABAergic synaptic function in a calpain-dependent mechanism. J. Biol. Chem. 288, 9634-9647 (2013).

19. Bannai, H. et al. Activity-dependent tuning of inhibitory neurotransmission based on GABAAR diffusion dynamics. Neuron 62, 670-682 (2009).

20. Loebrich, S., Bahring, R., Katsuno, T., Tsukita, S. \& Kneussel, M. Activated radixin is essential for GABAA receptor alpha5 subunit anchoring at the actin cytoskeleton. EMBO J. 25, 987-999 (2006).

21. Hausrat, T. J. et al. Radixin regulates synaptic GABAA receptor density and is essential for reversal learning and short-term memory. Nat. Commun. 6, 6872 (2015).

22. Wierenga, C. J. et al. Molecular and electrophysiological characterization of GFP-expressing CA1 interneurons in GAD65-GFP mice. PLoS ONE 5, e15915 (2010).
23. Tretter, V. et al. The clustering of GABA(A) receptor subtypes at inhibitory synapses is facilitated via the direct binding of receptor alpha 2 subunits to gephyrin. J. Neurosci. 28, 1356-1365 (2008).

24. Lopez-Bendito, G. et al. Preferential origin and layer destination of GAD65GFP cortical interneurons. Cereb. Cortex 14, 1122-1133 (2004).

25. Pashut, T. et al. Patch-clamp recordings of rat neurons from acute brain slices of the somatosensory cortex during magnetic stimulation. Front. Cell Neurosci. 8, 145 (2014)

26. Donato, F., Rompani, S. B. \& Caroni, P. Parvalbumin-expressing basket-cell network plasticity induced by experience regulates adult learning. Nature 504, 272-276 (2013).

27. Ruediger, S. et al. Learning-related feedforward inhibitory connectivity growth required for memory precision. Nature 473, 514-518 (2011).

28. Gibson, J. R., Huber, K. M. \& Sudhof, T. C. Neuroligin-2 deletion selectively decreases inhibitory synaptic transmission originating from fast-spiking but not from somatostatin-positive interneurons. J. Neurosci. 29, 13883-13897 (2009).

29. Becker, D. et al. Functional and structural properties of dentate granule cells with hilar basal dendrites in mouse entorhino-hippocampal slice cultures. PLoS ONE 7, e48500 (2012).

30. Gersner, R., Kravetz, E., Feil, J., Pell, G. \& Zangen, A. Long-term effects of repetitive transcranial magnetic stimulation on markers for neuroplasticity: differential outcomes in anesthetized and awake animals. J. Neurosci. 31, $7521-7526$ (2011).

31. Marder, E. \& Goaillard, J. M. Variability, compensation and homeostasis in neuron and network function. Nat. Rev. Neurosci. 7, 563-574 (2006).

32. Nelson, S. B. \& Turrigiano, G. G. Strength through diversity. Neuron 60, 477-482 (2008).

33. Hulme, S. R., Jones, O. D. \& Abraham, W. C. Emerging roles of metaplasticity in behaviour and disease. Trends Neurosci. 36, 353-362 (2013).

34. Long, Z. et al. Decreased GABA levels in anterior cingulate cortex/medial prefrontal cortex in panic disorder. Prog. Neuropsychopharmacol. Biol. Psychiatry 44, 131-135 (2013).

35. Ziemann, U., Ilic, T. V. \& Jung, P. Long-term potentiation (LTP)-like plasticity and learning in human motor cortex--investigations with transcranial magnetic stimulation (TMS). Suppl. Clin. Neurophysiol. 59, 19-25 (2006).

36. Benali, A. et al. Theta-burst transcranial magnetic stimulation alters cortical inhibition. J. Neurosci. 31, 1193-1203 (2011).

37. Murakami, T., Muller-Dahlhaus, F., Lu, M. K. \& Ziemann, U. Homeostatic metaplasticity of corticospinal excitatory and intracortical inhibitory neural circuits in human motor cortex. J. Neurophysiol. 590, 5765-5781 (2012).

38. Hamada, M., Murase, N., Hasan, A., Balaratnam, M. \& Rothwell, J. C. The role of interneuron networks in driving human motor cortical plasticity. Cereb. Cortex 23, 1593-1605 (2013).

39. Flores, C. E. et al. Activity-dependent inhibitory synapse remodeling through gephyrin phosphorylation. Proc. Natl Acad. Sci. USA 112, E65-E72 (2014).

40. Sola, M. et al. Structural basis of dynamic glycine receptor clustering by gephyrin. EMBO J 23, 2510-2519 (2004).

41. Saiyed, T. et al. Molecular basis of gephyrin clustering at inhibitory synapses: role of G- and E-domain interactions. J. Biol. Chem. 282, 5625-5632 (2007).

42. Herweg, J. \& Schwarz, G. Splice-specific glycine receptor binding, folding, and phosphorylation of the scaffolding protein gephyrin. J. Biol. Chem. 287, 12645-12656 (2012).

43. Meyer, G., Kirsch, J., Betz, H. \& Langosch, D. Identification of a gephyrin binding motif on the glycine receptor beta subunit. Neuron 15, 563-572 (1995)

44. Tretter, V. et al. Molecular basis of the gamma-aminobutyric acid A receptor alpha3 subunit interaction with the clustering protein gephyrin. J. Biol. Chem. 286, 37702-37711 (2011).

45. Meier, J., Vannier, C., Serge, A., Triller, A. \& Choquet, D. Fast and reversible trapping of surface glycine receptors by gephyrin. Nat. Neurosci. 4, 253-260 (2001).

46. Jacob, T. C. et al. Gephyrin regulates the cell surface dynamics of synaptic GABAA receptors. J. Neurosci. 25, 10469-10478 (2005).

47. Levi, S. et al. Homeostatic regulation of synaptic GlyR numbers driven by lateral diffusion. Neuron 59, 261-273 (2008).

48. $\mathrm{Wu}$, X. et al. gamma-Aminobutyric acid type A (GABAA) receptor alpha subunits play a direct role in synaptic versus extrasynaptic targeting. J. Biol. Chem. 287, 27417-27430 (2012).

49. Maric, H. M. et al. Molecular basis of the alternative recruitment of GABAA versus glycine receptors through gephyrin. Nat. Commun. 5, 5767 (2014).

50. Calamai, M. et al. Gephyrin oligomerization controls GlyR mobility and synaptic clustering. J. Neurosci. 29, 7639-7648 (2009).

51. Tyagarajan, S. K., Ghosh, H., Harvey, K. \& Fritschy, J. M. Collybistin splice variants differentially interact with gephyrin and $\mathrm{Cdc} 42$ to regulate gephyrin clustering at GABAergic synapses. J. Cell Sci. 124, 2786-2796 (2011).

52. Maas, C. et al. Synaptic activation modifies microtubules underlying transport of postsynaptic cargo. Proc. Natl Acad. Sci. USA 106, 8731-8736 (2009). 
53. Marsden, K. C., Shemesh, A., Bayer, K. U. \& Carroll, R. C. Selective translocation of $\mathrm{Ca} 2+/$ calmodulin protein kinase IIalpha (CaMKIIalpha) to inhibitory synapses. Proc. Natl Acad. Sci. USA 107, 20559-20564 (2010).

54. Kirsch, J. \& Betz, H. Glycine-receptor activation is required for receptor clustering in spinal neurons. Nature 392, 717-720 (1998).

55. Dejanovic, B. et al. Exonic microdeletions of the gephyrin gene impair GABAergic synaptic inhibition in patients with idiopathic generalized epilepsy. Neurobiol. Dis. 67, 88-96 (2014).

56. Colonnier, M. in The Organization of the Cerebral Cortex (eds Schmitt, F. O., Worden, F. G., Adelman, G. \& Dennis, S. G.) 125-152 (MIT Press, 1981).

57. Nguyen, R. et al. Parvalbumin and GAD65 interneuron inhibition in the ventral hippocampus induces distinct behavioral deficits relevant to schizophrenia. J. Neurosci. 34, 14948-14960 (2014).

58. Donato, F., Chowdhury, A., Lahr, M. \& Caroni, P. Early- and late-born parvalbumin basket cell subpopulations exhibiting distinct regulation and roles in learning. Neuron 85, 770-786 (2015).

59. Caroni, P., Chowdhury, A. \& Lahr, M. Synapse rearrangements upon learning: from divergent-sparse connectivity to dedicated sub-circuits. Trends Neurosci. 37, 604-614 (2014).

60. Pell, G. S., Roth, Y. \& Zangen, A. Modulation of cortical excitability induced by repetitive transcranial magnetic stimulation: influence of timing and geometrical parameters and underlying mechanisms. Prog. Neurobiol. 93, 59-98 (2011).

61. Lopez-Alonso, V., Cheeran, B., Rio-Rodriguez, D. \& Fernandez-Del-Olmo, M. Inter-individual variability in response to non-invasive brain stimulation paradigms. Brain Stimul. 7, 372-380 (2014).

62. Thielscher, A. \& Kammer, T. Electric field properties of two commercial figure8 coils in TMS: calculation of focality and efficiency. Clin. Neurophysiol. 115, 1697-1708 (2004).

63. Gabriel, C., Gabriel, S. \& Corthout, E. The dielectric properties of biological tissues: I. Literature survey. Phys. Med. Biol. 41, 2231-2249 (1996).

64. Opitz, A., Windhoff, M., Heidemann, R. M., Turner, R. \& Thielscher, A. How the brain tissue shapes the electric field induced by transcranial magnetic stimulation. NeuroImage 58, 849-859 (2011).

65. Thielscher, A., Opitz, A. \& Windhoff, M. Impact of the gyral geometry on the electric field induced by transcranial magnetic stimulation. NeuroImage 54, 234-243 (2011).

66. Plonsey, R. \& Heppner, D. B. Considerations of quasi-stationarity in electrophysiological systems. Bull. Math Biophys. 29, 657-664 (1967).

67. Windhoff, M., Opitz, A. \& Thielscher, A. Electric field calculations in brain stimulation based on finite elements: an optimized processing pipeline for the generation and usage of accurate individual head models. Hum. Brain Mapp. 34, 923-935 (2013).

68. Radman, T., Ramos, R. L., Brumberg, J. C. \& Bikson, M. Role of cortical cell type and morphology in subthreshold and suprathreshold uniform electric field stimulation in vitro. Brain Stimul. 2, 215-228, e1-3 (2009).
69. Vlachos, A. et al. Synaptopodin regulates denervation-induced homeostatic synaptic plasticity. Proc. Natl Acad. Sci. USA 110, 8242-8247 (2013).

70. Nusser, Z. \& Mody, I. Selective modulation of tonic and phasic inhibitions in dentate gyrus granule cells. J. Neurophysiol. 87, 2624-2628 (2002).

71. Pfaffl, M. W. A new mathematical model for relative quantification in real-time RT-PCR. Nucleic Acids Res. 29, e45 (2001).

\section{Acknowledgements}

We thank Charlotte Nolte-Uhl and Nadine Zahn for their skillful assistance in tissue culturing, and Drs Heinrich Betz and Theofilos Papadopoulos for helpful discussions. This work was supported by Paul and Ursula Klein-Foundation, Dr Paul and Cilli WeillFoundation (both to A.V.), by Deutsche Forschungsgemeinschaft (FOR1332, CRC1080 to T.D. and A.V.) and by the Federal Ministry of Education and Research, Germany (BMBF; GCBS-WP1 to K.F. and A.V.).

\section{Author contributions}

The study was conceived and supervised by A.V. Experiments were designed by M.L., T.D., K.F. and A.V. Data were acquired and analysed by M.L., C.G., A.V. Computational modelling was carried out by A.O. The manuscript was written by A.V. with the help of M.L. and T.D. F.M.-D., C.J.W., G.S. and U.Z. were involved in data interpretation and critically revising the manuscript.

\section{Additional information}

Supplementary Information accompanies this paper at http://www.nature.com/ naturecommunications

Competing financial interests: U.Z. has received honoraria from Biogen Idec Deutschland GmbH, Bayer Vital GmbH, CorTec GmbH, Medtronic and Servier for advisory work. The remaining authors declare no competing financial interests.

Reprints and permission information is available online at http://npg.nature.com/ reprintsandpermissions/

How to cite this article: Lenz, M. et al. Repetitive magnetic stimulation induces plasticity of inhibitory synapses. Nat. Commun. 7:10020 doi: 10.1038/ncomms10020 (2016).

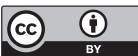

This work is licensed under a Creative Commons Attribution 4.0 International License. The images or other third party material in this article are included in the article's Creative Commons license, unless indicated otherwise in the credit line; if the material is not included under the Creative Commons license, users will need to obtain permission from the license holder to reproduce the material. To view a copy of this license, visit http://creativecommons.org/licenses/by/4.0/ 